\title{
Nikolaus Straub von Leonberg (um 1415-um 1500) - Notar und Bibelübersetzer
}

\author{
Von Andreas Deutsch
}

Eher unscheinbar wirkt Manuskript Nr. 35 der Leipziger Universitätsbibliothek. Dass es sich hierbei um eine - jedenfalls deutlich vor Luther entstandene - Übersetzung der vier Evangelien des Neuen Testaments (samt der Vorreden des Hieronymus) handelt, ist in der Wissenschaft längst bekannt, doch sonst ist fast alles umstritten. Die in dieser einzigen Handschrift überlieferte undatierte Bibelübersetzung endet mit den Worten: Almechtiger got verlieh uns ein guit end [...] Nicolaus Straub sst. [?] notarius hallensis. Nur hierdurch ist der Name des Übersetzers bekannt, der sich zugleich selbst als Notar von Hall(e) ausweist. Unsicher war allerdings bislang, welches Hall(e) damit gemeint ist. Während die ältere Forschung für Schwäbisch Hall plädierte, nahm die moderne Forschung bislang eine Entstehung im „ostmitteldeutsch-baierischen Grenzgebiet“ an. Da das Manuskript heute in Leipzig liegt, schien eine Entstehung in Halle an der Saale plausibel. Allerdings kam das Manuskript erst im 19. Jahrhundert aus unbekannter Herkunft nach Leipzig. Auch in Bezug auf die sprachliche Zuordnung sind erhebliche Zweifel angebracht.

\section{A. Das Manuskript}

\section{Forschungsstand}

Als erster wies Erich Zimmermann in einem kurzen Beitrag aus dem Jahre 1939 auf die Handschrift hin. Aufgrund sprachlicher Kriterien hielt er eine Entstehung in Schwäbisch Hall für wahrscheinlich ${ }^{1}$. Dieser Hypothese schloss sich Karl Langosch 1955 im „Verfasserlexikon“ unbesehen an². Für den Genealogen und Sozial-

${ }^{1}$ Erich Zimmermann, Die Leipziger Handschrift Ms. 35, in: Neue Forschungen und Texte zur Geschichte der deutschen Bibel, hg. von Hans Vollmer (Bibel und deutsche Kultur, Bd. 9), Potsdam 1939, S. 70*-76*.

2 Karl Langosch, Art. „Straub, Nicolaus“, in: Die deutsche Literatur des Mittelalters Verfasserlexikon, hg. von Wolfgang STAmmLeR, Bd.5, Berlin ${ }^{1}$ 1955, Sp. 1069. Vorsichtiger: Stefan Sonderegger, Geschichte deutschsprachiger Bibelübersetzungen in Grundzügen, in: Sprachgeschichte - ein Handbuch zur Geschichte der deutschen Sprache und ihrer 
historiker Gerd Wunder schließlich bestanden keinerlei Zweifel, dass die Übersetzung in Schwäbisch Hall angefertigt wurde; er hatte zwar das Manuskript selbst nicht konsultiert, verwies in seinen „Bürgern von Hall“ 1980 aber auf den Umstand, dass ein Notar mit Namen Nikolaus Straub in Schwäbisch Hall nachzuweisen ist ${ }^{3}$.

In der neueren Forschungsliteratur setzte sich demgegenüber eine andere Position durch: So verlegte Heimo Reinitzer in der 1995 erschienenen Neuauflage des Verfasserlexikons die Übersetzung aufgrund ihrer Sprache ins „ostmitteldeutschbaierische Grenzgebiet“4. Dieser „Analyse“ schließt sich Reinhard Müller 2000 in der Neuauflage des „Deutschen Literatur-Lexikons“ an; er meint - vermutlich unter Bezugnahme auf Wunder -, der Verfasser „sei nicht mit Sicherheit identifizierbar" ${ }^{\text {"5 }}$. Ohne auf den Verfasser überhaupt einzugehen, halten Pensel und Stahl in ihrem Verzeichnis der Leipziger mittelalterlichen Handschriften „Straubs Evangeliar" für schwäbisch mit baierischen Elementen", während Stefan Sonderegger vorsichtiger von „oberdeutscher Sprache“ schreibt ${ }^{7}$.

Auf der Basis dieser Fakten war bislang naturgemäß auch keine Datierung des Werkes möglich ${ }^{8}$. Die Literatur schließt sich Langosch an, der Straubs Bibel vorsichtig ins 15. Jahrhundert setzt. Dass eine Übersetzung ohne sicheren Verfasser, ohne Entstehungsdatum und ohne Entstehungsort bislang nicht gerade die Neugier der Bibelforscher anregte, verwundert kaum. Hinzu kommt, dass die Handschrift zu Zeiten der DDR kaum zugänglich war. Der nachfolgende Beitrag möchte die Evangelien-Übersetzung dem Vergessen entreißen. Eine Neubewertung der Fakten soll den Nachweis erbringen, dass das Werk in Schwäbisch Hall entstanden ist. Schließlich soll eine biographische Skizze die Person Nikolaus Straubs näherbringen - denn immerhin erscheint es ungewöhnlich, dass sich ein Notar an das Übersetzen der vier Evangelien macht.

Erforschung, hg. von Werner BEsCH u. a. (Handbücher zur Sprach- und Kommunikationswissenschaft, Bd.2.1), Berlin/New York 21998, S. 229-284, hier S. 256.

${ }^{3}$ Gerd Wunder, Die Bürger von Hall - Sozialgeschichte einer Reichsstadt 1216-1802, Sigmaringen 1980, S.123. Er beruft sich laut S. 310 auf: Bibel und deutsche Kultur 9, 1939, S. 70 .

${ }^{4}$ Heimo Reinitzer, Art. „Straub, Nicolaus“, in: Die deutsche Literatur des Mittelalters - Verfasserlexikon, hg. von Kurt RuH u. a., Bd. 9, Berlin/New York 21995, Sp. 386 f.

${ }^{5}$ Reinhard Müller, Art. „Straub, Nicolaus“, in: Deutsches Literatur-Lexikon, Bd. 20, hg. von Hubert Herkommer/Konrad Feilchenfeldt, Zürich/München ${ }^{32000, ~ S p . ~} 523$.

${ }^{6}$ Franzjosef Pensel, Verzeichnis der deutschen mittelalterlichen Handschriften in der Universitätsbibliothek Leipzig, zum Druck gebracht von Irene STAHL (Deutsche Texte des Mittelalters, Bd.70,3), Berlin 1998, S.6f.

7 Sonderegger (wie Anm. 2) S. 256.

8 Vgl. allerdings den diesem Beitrag vorgreifenden Artikel: Andreas Deutsch, „Straub(e), Nicolaus (auch Strub, Struwe) von Le(o)nberg" [Nikolaus Straub, Bibelübersetzer und Notar], in: Neue Deutsche Biographie, Bd.25, hg. von der Historischen Kommission bei der Bayerischen Akademie der Wissenschaften, Berlin 2013, S. $488 \mathrm{f}$. 


\section{Schlusssatz}

Den wichtigsten Hinweis auf Verfasser, Entstehungsort und -zeit liefert in der Tat der bereits erwähnte Schlusssatz der Handschrift, der im vollen Wortlaut wie folgt lautet:

Almechtiger got verlieh uns ein guit end - Subicio me correctioni melius transferencium, de nudo enim textu transtuli glosas non videns - Nicolaus Straub sst. $\left[\right.$ ? - u. a. gedeutet als: subscripsit $\left.{ }^{9}\right]$ notarius hallensis ${ }^{10}$.

Es ist dies der einzige lateinische Satz des fast vierhundert Seiten starken Buches. Der Übersetzer fordert hierin zur Korrektur seines Textes auf, denn er habe ihn „aus dem nichts“, also ohne Vorlagen oder irgendwelche Glossen, ins Deutsche übertragen. Anschließend unterzeichnet er mit Nikolaus Straub, Notar von Hall. Nun gibt es in der Tat viele „Hall“; und bei einer Lagerung der Handschrift in Leipzig könnte man zunächst an Halle an der Saale denken. Die Handschrift der „Straubbibel“ ist allerdings aus ungeklärter Herkunft erst im 19. Jahrhundert in die Leipziger Universitätsbibliothek gelangt ${ }^{11}$. Sie ist in einem braunen Ledereinband mit Holzdeckeln und eisernen Buckeln gefasst. Interessant ist vielleicht, dass zur Verstärkung des Einbands inwendig zwei zusammengehörige Pergamentblätter einer liturgischen Schrift wohl des frühen 12. Jahrhunderts wiederverwertet wurden, die unter anderem die Heiligenlegenden von Sankt Kilian und Sankt Felicitas enthalten ${ }^{12}$. Bekanntlich wurde Kilian besonders in Franken verehrt, zu denken ist beispielsweise an die Kilianskirche in Heilbronn. Ähnliches gilt eingeschränkt für die Heilige Felicitas ${ }^{13}$.

Weiter kommt man, wenn man sich an den - durch die Unterschrift gesicherten Namen - des Verfassers hält. Der Name Nikolaus Straub (natürlich in allen Schreibvarianten, z. B. Strube, Strupp, Struwe, Straube) lässt sich in der fraglichen Zeit (15. Jahrhundert) nämlich von allen „Hall“ ausschließlich in Schwäbisch Hall nachweisen. Aufgrund der Lückenhaftigkeit der Quellenlage im 15. Jahrhundert ist dies allein freilich kein Beweis, zumal die sprachliche Analyse der neueren Wissenschaft diesem Ergebnis zu widersprechen scheint.

9 U.a. von Zimmermann (wie Anm.1) S.70*, gedeutet als: „subscripsit“; Rudolf Helssig (Die lateinischen und deutschen Handschriften der Universitätsbibliothek Leipzig, Bd.1: Die theologischen Handschriften, Teil 1 (Ms 1-500), Leipzig 1926, Nachdruck Wiesbaden 1995, S.36f., hier S.37) liest: „publicus“, was ebenso zweifelhaft erscheint wie „proto-“ im Verfasserlexikon. Subscripsit vermutet auch Dr. Harald Drös, Leiter der Inschriftenkommission bei der Heidelberger Akademie der Wissenschaften (Herzlichen Dank für die Auskunft!).

${ }^{10}$ Universitätsbibliothek Leipzig, Ms 35, Bl. $176 \mathrm{v}$.

11 Hierzu Helssig (wie Anm. 9) S.36f.

12 Ebd., S.36.

${ }^{13}$ Lambert DörR, Zur Verehrung der heiligen Felizitas in der mittelalterlichen Abtei Münsterschwarzach, in: Mainfränkisches Jahrbuch 17 (1965) S. 17-28. 


\section{Sprachliche Beurteilung und Schriftvergleich}

Möglicherweise standen den Sprachwissenschaftlern als Grundlage für ihre geographische Zuordnung allein jene bescheidenen Ausschnitte aus der „Straubbibel“ zur Verfügung, die am Ende des Beitrags von Zimmermann und in der Sammlung biblischer Übersetzungshandschriften von Splett abgedruckt sind ${ }^{14}$. Bei einem ausführlicheren Blick in den Text erscheinen typisch mitteldeutsche Sprachelemente jedenfalls kaum erkennbar, während der Text insgesamt süddeutsch wirkt ${ }^{15} . \mathrm{Zu}$ einem übereinstimmenden Ergebnis führte ein Abgleich häufiger und typischer Schreibformen aus den Straub-Evangelien mit der Datenbank des Deutschen Rechtswörterbuchs (DRW ${ }^{16}$. Da die Belege des DRW jeweils mit Regionalangaben versehen oder über ihre Siglenbezeichnung regional zuordenbar sind, lässt sich über eine solche Suche feststellen, in welcher Region bzw. welchen Regionen Belege mit der in der „Straubbibel“ vorgefundenen Schreibform nachweisbar sind. Zweifellos muss bei diesem Verfahren mit einer gewissen Unschärfe gerechnet werden, schließlich kann auch ein breit angelegtes Wörterbuch nicht immer alle Schreibformen eines Wortes abbilden; insgesamt ergab sich jedoch ein unerwartet einheitliches Bild: Neben zahlreichen Schreibformen die allgemein süddeutsch sind (also auch für das Bairische oder Alemannische typisch sind, etwa habend, küng, wiss), enthält der Text auch solche, die im 15. Jahrhundert nur in Schwaben belegt sind (z. B. tond für „sie tun“ und tüwe für „tue“, aubent für Abend, schlauff für Schlaf, floub für „flüchtete“) - und dieses schwäbische Element überwiegt deutlich, auch wenn es daneben gewisse bairische oder fränkische Einflüsse zu geben scheint.

Es ist somit nicht mehr allzu abwegig, an den in Schwäbisch Hall nachweisbaren Nikolaus Straub als Bibelübersetzer zu denken. Da Straub Notar war, hat er glücklicherweise eine mehr als hinreichende Anzahl von Urkunden aus eigener Feder hinterlassen, die mit dem Bibelmanuskript verglichen werden können. Hierbei fällt zunächst auf, dass die eben beschriebenen typischen Schreibformen der „Straubbibel“ (habend, tond usw.) auch in den von Straub verfertigten Urkunden begegnen. Auch die Handschrift weist derart markante Ähnlichkeiten auf, dass dies deutlich über die typischen Merkmale einer geübten Kanzleischrift hinausgeht.

Den Beweis aber, dass der Evangelien-Übersetzer mit dem Schwäbisch Haller Notar identisch ist, liefert die Unterschrift, denn nicht nur die „Straubbibel“ ist unterschrieben: Aufgrund ihrer Beweisfunktion musste jede formal korrekt ausgestellte Notarsurkunde der Zeit neben dem Notarssignet (also einem wappenartigen

14 Vgl. hierzu unten in Anm. 157.

15 Freundlicher Hinweis der Sprachwissenschaftlerin Eva Lill von der Forschungsstelle Deutsches Rechtswörterbuch.

${ }^{16}$ Ein Großteil der Datenbank ist über www.deutsches-rechtswoerterbuch.de auch online abrufbar; Schreibformen lassen sich dort über die Suchfunktion „Schreibformen“ abfragen. 
gezeichneten „Logo“ des Notars ${ }^{17}$ ) eine saubere und präzise Unterschrift des jeweiligen Notars enthalten. Die Unterschrift auf Straubs Urkunden stimmen exakt mit jener am Ende der „Straubbibel“ überein.

Hier einige Beispiele zum Vergleich:

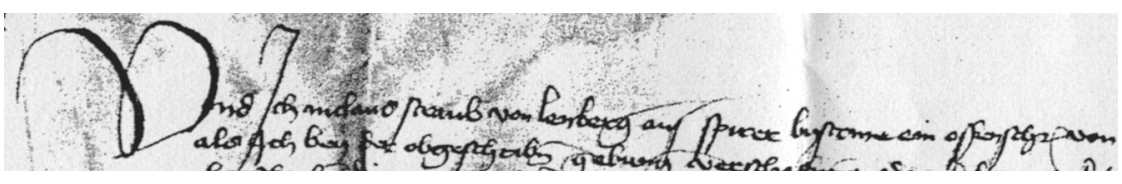

Abb.1: Unterschrift von Nikolaus Straub auf einer Urkunde vom 1. März 1463 (StAL B 186 U 1254).

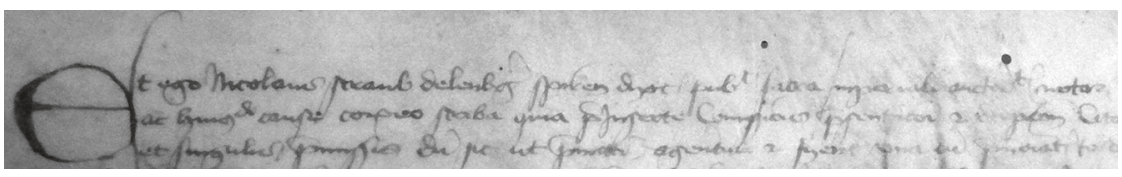

Abb. 2: Unterschrift von Nikolaus Straub auf einer lateinischen Urkunde von 1465 (eingebunden in: Nikolaus Straub, handschriftlicher Institutionenkommentar, Landesbibliothek Speyer, Nr. 1).

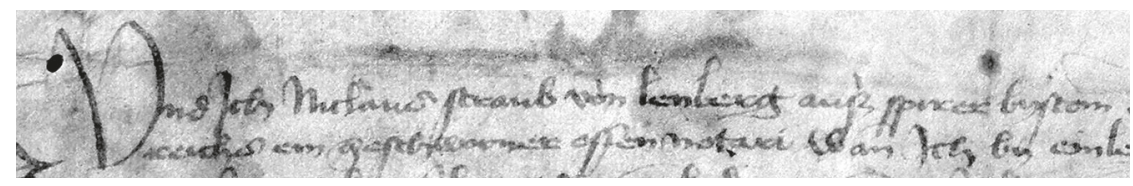

Abb. 3: Unterschrift von Nikolaus Straub auf einer deutschen Urkunde von 1467 (eingebunden in: Nikolaus Straub, handschriftlicher Institutionenkommentar, Landesbibliothek Speyer, Buch Nr. 1).

17 In der Regel waren Notare nicht berechtigt, ein Siegel zu führen, als „Freischaffende“ waren sie auch nicht berechtigt, das Siegel der Stadt oder des Landes zu führen, in dem sie tätig waren. Gewissermaßen als Ersatz hierfür bestand in Deutschland der Brauch, dass die Notare Signete, in gewisser Weise gezeichnete Siegel - mit ebenderen Funktion, führten. Jeder Notar konnte sich sein Signet nach freiem Belieben wählen, manche Notare entwickelten hierbei ein geradezu erstaunliches zeichnerisches Geschick. Vgl. etwa: Wilhelm Sснміdт-Thомé, Die Bestellung zum Notar im altdeutschen Recht, in: Deutsche NotarZeitschrift 1973, S. 261 -275, hier S. 265. Vgl. auch Art. „Signet“ im Deutschen Rechtswörterbuch, Bd.13, Sp. $561 \mathrm{f}$. 

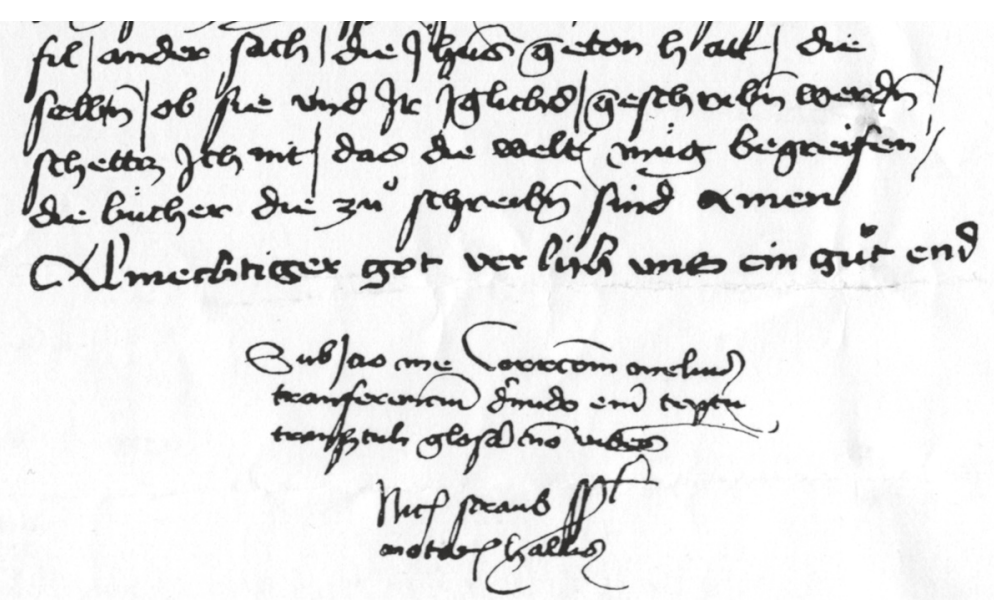

Abb. 4: Unterschrift von Nikolaus Straub am Ende der Evangelien-Übersetzung (Universitätsbibliothek Leipzig, Ms 35).

\section{B. Aus dem Leben des Nikolaus Straub}

\section{Herkunft und Ausbildung}

Straub führte oft als Beinamen von Lenberg Spirer Bistums, manchmal liest man aber auch Nikolaus Straub von Leuberg ${ }^{18}$, Nycolaus Strube de Leomberg oder lateinisch Nycolaus Strube de Leuwenberch ${ }^{19}$ bzw. Nicolaus Straub de Lemberg Spirensis dyocesis ${ }^{20}$. Das hinter dem Ortsnamen angefügte Spirer Bistums, Spirensis dyocesis usw. wird zumeist als Präzisierung dieser Herkunftsangabe gelesen. Auf einer späten Urkunde - vom 5. Dezember 1498 - unterzeichnet Straub allerdings als nicolaus straub de lenberg clericus coningatus spirensis dyocesis ${ }^{21}$; er war dem-

18 Landesarchiv Baden-Württemberg, StAL B 375 S U 452.

19 Liber I Actorum Facvltatis Artivm Academiae Heidelbergensis, Bl. 86 r., im Universitätsarchiv Heidelberg, Signatur H IV 101/1; vgl. auch Dagmar DrüLL, Heidelberger Gelehrtenlexikon 1386-1651, Berlin/Heidelberg 2002, S. 410.

20 Vgl. U 820 in: Urkundenbuch der Stadt Heilbronn: Bd.1 (bis 1475) (Württembergische Geschichtsquellen, Bd.5), bearb. von Eugen Knupfer, Stuttgart 1904; Bd.2 (1476-1500) (Württembergische Geschichtsquellen, Bd.15), bearb. von Moriz von Rauch, Stuttgart 1913; Bd.3 (1501-1524) (Württembergische Geschichtsquellen, Bd.19), bearb. von Moriz vON RaUch, Stuttgart 1916 (nachfolgend UB Heilbronn).

${ }^{21}$ Landesarchiv Baden-Württemberg, StAL B 189 III U 63; für das Auffinden danke ich herzlich Prof. Dr. Maria Magdalena Rückert vom dortigen Archiv. Vgl. auch die Urkunde vom 8. April 1444 in: UB Heilbronn (wie Anm. 20) U 631, die laut Regest einen ähnlichen Vermerk hat. 
zufolge verheirateter Kleriker, also ein Geistlicher mit niederen Weihen, der Speyerer Diözese ${ }^{22}$. Man könnte nun aufgrund dieser Formulierung annehmen, dass auch alle anderen Erwähnungen der Speyerer Diözese hinter dem Namen als verkürzter Hinweis auf Straubs im Speyerer Bistum erworbenen niederen Weihen zu lesen sind, zumal es im 15. Jahrhundert durchaus verbreitet war, dass Notare auf ihre Eigenschaft als Kleriker unter Angabe des Bistums verwiesen ${ }^{23}$. Eine Präzisierung des Herkunftsorts durch Nennung der Diözese, in welcher dieser liegt, war indes mindestens ebenso üblich. Wenn das Bistum des Herkunftsorts und jenes der niederen Weihen auseinanderfielen, wurde bisweilen sogar beides genannt, so heißt es in einer Otterberger Urkunde: Et ego Fridericus Pawini de Andernaco, Treverensis dyecesis, clericus coningatus Spirensis publicus auctoritate imperiali et prefati domini prepositi notarius ${ }^{24}$. Dass Straub mit seiner Ergänzung der Ortsangabe mit Spirer Bistums, Spirensis dyocesis usw. tatsächlich den Ort seiner Herkunft präzisieren wollte, ergibt sich im Übrigen auch zweifelsfrei aus der Formulierung einzelner Urkunden, wenn es etwa heißt: Niclaus straub von lenberg auß spirer bystom ${ }^{25}$.

Man hat daher vermutet ${ }^{26}$, Straub stamme aus dem bei Pirmasens gelegenen Lemberg in der Pfalz. Die 4.000-Einwohner-Gemeinde gehört heute nämlich zum Bistum Speyer. Dies war allerdings bis weit ins 19. Jahrhundert hinein anders: Zusammen mit Pirmasens, Rodalben, Clausen und Schloss Gräfenstein lag das im Mittelalter nicht ganz unbedeutende Lemberg im Gebiet der Diözese Metz ${ }^{27}$. Blickt man hingegen nach Leonberg, das heute zum Bistum Rottenburg-Stuttgart zählt und auf historischen Landkarten oft der Konstanzer Diözese zugeschlagen

${ }^{22}$ Vermutlich dem Regesteneintrag folgend bezeichnet auch Schuler Straub als Kleriker. Vgl. Peter-Johannes ScHuler, Notare Südwestdeutschlands - ein prosopographisches Verzeichnis für die Zeit von 1300 bis ca. 1500, Textband, Stuttgart 1987, Nr.1326, S. 450.

${ }^{23}$ Vgl. nur: Johann Gustav Gottlieb Büsching, De signis seu signetis notariorum veterum in Silesiacis tabulis, Breslau 1820, z. B. U 64 (1407): Franciscus Hertelin de Sagano clericus conjugatus Wratisl. dioc. jmperiali auctoritate publicus notarius; U 81 (1440): Bartholomei Grädis de Maioriglonia wrat. dioc. Clericus Coningatus publicus Imperiali auctoritate Notarius; ferner: Bernhard TönNIEs (Hg.), Die Handschriften der Thüringer Universitätsund Landesbibliothek Jena I, Wiesbaden 2002, Ms. El. f. 37 (1447): [Notar] Johannes Henrici de Reichinbach clericus coniugatus Misnensis dyocesis.

${ }^{24}$ Vgl. U 416 (1329) in: Urkundenbuch des Klosters Otterberg in der Rheinpfalz, hg. von Michael Frey/Franz Xaver Remling, Mainz 1845.

${ }^{25}$ Vgl. die dem Buch Nr. 1 in der Landesbibliothek Speyer beigebundene deutschsprachige Urkunde von 1467.

${ }^{26}$ Vgl. etwa die Einträge (und das Register) bei Friedrich PieTsCh (Bearb.), Die Urkunden des Archivs der Reichsstadt Schwäbisch Hall, Bd.1 (1156-1399) (Veröffentlichungen der Staatlichen Archivverwaltung Baden-Württemberg, Bd.21), Stuttgart 1967; Bd.2 (1400-1479) (Veröffentlichungen der Staatlichen Archivverwaltung Baden-Württemberg, Bd.22), Stuttgart 1972 (nachfolgend zitiert als UB Schwäbisch Hall).

27 Franz Xaver Remling, Geschichte der Bischöfe zu Speyer, Bd.1, Mainz 1852, S. 109; Michael FrEY, Versuch einer geographisch-historisch-statistischen Beschreibung des königlich bayerischen Rheinkreises, dermalen Pfalz, Bd.4, Speyer 1837, S. 270 f. 
wird, so stellt man fest, dass das Städtchen im Mittelalter tatsächlich zum Bistum Speyer gehörte ${ }^{28}$. Lenberg Speyerer Bistums steht somit für Leonberg ${ }^{29}$.

Da sich in Leonberg kaum Archivalien aus dem frühen 15. Jahrhundert erhalten haben, verwundert nicht, dass Straubs Kindheit und frühe Jugend gänzlich im Dunkeln liegen, sich auch über seine Familie in Leonberg nichts ermitteln lässt ${ }^{30}$. Er muss eine gute Schulbildung genossen haben, vielleicht in einer Klosterschule, wo er bereits seine niederen Weihen erworben haben könnte. Zu Beginn seines Studiums lässt sich dann Straubs Spur aufnehmen: In den am 20. Dezember 1431 ausgelegten Matrikeln der Universität Heidelberg schrieb sich Nycolaus Strub de Leonberg Spir. dyoc. ein ${ }^{31}$. Hieraus lässt sich auf eine Geburt um das Jahr 1415 rückschließen, denn wesentlich jünger als 15 Jahre alt wird er bei seiner Immatrikulation kaum gewesen sein - ein wesentlich höheres Alter erscheint aufgrund seines weiteren Lebenslaufs unwahrscheinlich ${ }^{32}$.

Bekanntermaßen ist die 1386 gegründete Heidelberger Universität die älteste auf dem Boden der Bundesrepublik Deutschland, eine große Studienort-Auswahl hatte Straub, als er 1431 sein Studium begann, noch nicht. Immerhin gab es neben Prag (gegründet 1348) und Wien (1365) im deutschsprachigen Raum dann auch Universitäten in Köln (1388), Erfurt (1392), Würzburg (1402), Leipzig (1409) und Rostock (1419). Sieht man einmal von der recht kurzlebigen Würzburger Gründung ab, hätte Straub zu jeder dieser Universitäten einen erheblich weiteren Reiseweg als nach Heidelberg gehabt, es wundert also nicht, dass Straub gerade in den

28 „Lewenberg“ im Dekanat „Grienningen“, heute Markgröningen, im Archidiakonat Allerheiligen zu Speyer, vgl. die auf einem Verzeichnis von 1482 beruhende Auflistung bei: Friedrich Thudichum, Die Diözesen Konstanz, Augsburg, Basel, Speier, Worms nach ihrer alten Einteilung in Archidiakonate, Dekanate und Pfarreien, Tübingen 1906, S. 111 ff., insbesondere S. 118.

${ }^{29}$ So auch fast die gesamte neuere Literatur, vgl. bereits die Regesteneinträge von Eugen KNupfer und Moriz von RAuch in: UB Heilbronn (wie Anm. 20); vgl. ferner etwa WundeR (wie Anm.3) S.123; Alexander Brunotte/Raimund J. Weber (Bearb.), Akten des Reichskammergerichts im Hauptstaatsarchiv Stuttgart: Inventar des Bestands C 3, Bd. 3, Stuttgart 1999, S. 426 f.; Deutsch, „Straub(e), Nicolaus“ (wie Anm. 8) S. $488 \mathrm{f}$.

30 Freundliche Auskunft von Frau Stadtarchivarin Bernadette Gramm vom 27. Mai 2010.

31 Die Matrikel der Universität Heidelberg von 1386 bis 1662, bearb. und hg. von Gustav Toepke, Bd.1: Von 1386 bis 1553, Heidelberg 1884, S. 188.

32 Eine Immatrikulation mit 15 Jahren war damals nicht unüblich; vgl. etwa zur Einschreibung des späteren Reformators Johannes Brenz an der Heidelberger Artistenfakultät in diesem Alter (Joan. printz de weyla spiren. dioc.): Hermann EHMER, Johannes Brenz Herkunft und Ausbildung, in: Johannes Brenz 1499-1570: Prediger, Reformator, Politiker, Ausstellung im Hällisch-Fränkischen Museum Schwäbisch Hall und im Württembergischen Landesmuseum Stuttgart, Schwäbisch Hall 1999, S.36-43, 38f. Als typisches Alter für den Studienbeginn an der spätmittelalterlichen Erfurter Universität ermittelte Gramsch fünfzehn bis achtzehn Jahre, zumeist waren die Studienanfänger sechzehnjährig, bisweilen aber auch deutlich jünger, in einem Fall nur elf, vgl. Robert GRAmsch, Erfurter Juristen im Spätmittelalter - Karrieremuster und Tätigkeitsfelder, Leiden 2003, S. 189 f. 
Heidelberger Matrikeln - und nur dort - begegnet. Trotz bescheidener Anfänge bestanden in Heidelberg fast von Anfang an die klassischen vier Fakultäten: Artisten (Artes liberales, Philosophie), Medizin (Naturwissenschaften), Jura und Theologie ${ }^{33}$.

Dem mittelalterlichen Studienaufbau folgend, musste Straub - unabhängig von seinen Interessen - zunächst die Artistenfakultät besuchen. Er war offensichtlich ein eifriger Student, denn sein Examen als „Bakkalaureus“ legte er bereits am 18. Juli 1433, also nach anderthalb Jahren, $\mathrm{ab}^{34}$. Noch einmal zwei Jahre später - im Mai 1435 - absolvierte er das Magisterexamen der Artistenfakultät, wurde also „Magister artium“. In den Prüfungslisten heißt es: Nycolaus Strube de Leomberg. d. 2 1 1 2 fl., det. sub M. Alb. de Mulbusen 19. May - was zeigt, dass ein Examen damals eine kostspielige Angelegenheit war und Straub hinreichend Geld hatte. Der „Magister artium“ war in der ersten Hälfte des 15. Jahrhunderts ein durchaus beachtlicher Abschluss. Er bescheinigte Straub unter anderem jene vorzüglichen Lateinkenntnisse, die in seinen Schriften später sichtbar werden.

Im Anschluss an ein erfolgreich abgeleistetes Magisterexamen bestand in Heidelberg die Verpflichtung zu mindestens zweijähriger Lehrtätigkeit an der Fakultät ${ }^{35}$. Tatsächlich begegnet Straub nun als Mitarbeiter der Artistenfakultät. Möglicherweise diente ihm diese Tätigkeit auch zur Finanzierung eines Studiums an einer der drei höheren Fakultäten - eine um diese Zeit übliche Vorgehensweise ${ }^{36}$. Da Straub später als Notar wirkte, liegt der Gedanke nahe, er könnte wenigstens eine Zeitlang Jura studiert haben. Selbstverständlich oder gar notwendig war dies indes nicht; noch hundert Jahre später haben die meisten Männer, die in juristischen Berufen tätig waren, niemals eine Universität von innen gesehen, geschweige denn Jura studiert.

Im Juni 1437 wurde Nicolas strub de lemberg in das consilium facultatis artium, also den Fakultätsrat, gewählt ${ }^{37}$; er war demzufolge noch immer an der Artistenfakultät aktiv. Das zur Unterstützung und Beratung des Dekans eingesetzte Gremium war 1437 neu organisiert worden; hatte es zuvor aus nur zwei bis vier Mitgliedern bestanden, waren es nun zehn ${ }^{38}$. Offenbar erhielt Straub im Zuge dieser Neustrukturierung sein Amt als consiliarius facultatis. Nach 1437 verlieren sich Straubs Spuren in Heidelberg. Möglicherweise verließ er die Stadt aufgrund der dort ab November des Jahres wütenden Pest. Am 25. November erlaubte die

33 Eike Wolgast, Die Universität Heidelberg 1386-1986, Berlin/Heidelberg/New York 1986, S. 3.

${ }^{34}$ Die Matrikel der Universität Heidelberg (wie Anm. 31) Bd. 2, S. 383.

35 Wolgast (wie Anm. 33) S. 9.

36 Ebd.

37 Liber I Actorum Facvltatis Artivm Academiae Heidelbergensis, Bl. 86 r., in: Universitätsarchiv Heidelberg, Signatur H IV 101/1; hierzu: DrüLL (wie Anm. 19) S. 410.

38 Drüll (wie Anm. 19) Bd.3, Glossar, S. XXVII. 
Universität ihren Angehörigen sogar offiziell den Wegzug in sicherere Orte ${ }^{39}$, zu diesem Zeitpunkt dürfte bereits ein großer Teil der Universitätsmitglieder die Flucht ergriffen haben.

\section{Freier Notar und Schultheiß von Wimpfen im Tal}

Den nächsten Hinweis auf Straub bringt erst das Jahr 1444: Eine in einem Heilbronner Haus ausgefertigte Urkunde über eine Schenkung von Todes wegen stammt aus der Feder des Notars Nikolaus Strub von Leonberg ${ }^{40}$. Schon aufgrund des Namenszusatzes „von Leonberg“ besteht kein Zweifel, dass es sich hierbei um denselben Mann handelt; Straub ist demzufolge in der Zwischenzeit zum Notar avanciert.

Eine festgelegte Schulung oder gar eine bestimmte Abschlussprüfung gab es für die Notarslaufbahn damals noch nicht. Dennoch ist davon auszugehen, dass Straub, bevor er Notar werden konnte, erst einmal eine einschlägige Ausbildung genoss. Dafür gab es im Prinzip zwei Möglichkeiten: Zum einen konnte er eine der sogenannten „Schreiberschulen“ besuchen, die den Kanzleien der größeren Städte angeschlossen waren. Berühmt war beispielsweise die Schreiberschule des Nikolaus von Wyle, der ab zirka 1447 in Esslingen als Stadtschreiber (also Kanzleichef) fungierte ${ }^{41}$. Auch der Verfasser des bekannten Klagspiegels (um 1436), der Schwäbisch Haller Stadtschreiber Conrad Heyden, bildete Schreibschüler aus, die ihm zugleich als Assistenten dienten ${ }^{42}$. Zum anderen konnte sich Straub einem bereits tätigen Notar anschließen und als dessen Schüler sein „Handwerk“ erlernen.

Um den Beruf des Notars auszuüben, war dann aber die offizielle Ernennung entscheidend. Auch hier gab es zwei Möglichkeiten: Die Ernennung zum päpst-

39 U 290, in: Urkundenbuch der Universitaet Heidelberg, zur 500jährigen Stiftungsfeier der Universität, Bd.2, hg. von Eduard Winkelmann, Heidelberg 1886.

${ }^{40}$ UB Heilbronn (wie Anm. 20) U 631.

41 Etwa: Otto MaYer, Geistiges Leben in der Reichsstadt Eßlingen vor der Reformation der Stadt. Eine kulturgeschichtliche Studie, in: WVjH NF 9 (1900) S.1-32 und 311-367, insbesondere S.26; Rolf Schwenk, Vorarbeiten zu einer Biographie des Niklas von Wyle und $\mathrm{zu}$ einer kritischen Ausgabe seiner ersten Translatze, Göppingen 1978; Franz Josef Worstвrock, Art. „Niklas von Wyle“, in: Die deutsche Literatur des Mittelalters Verfasserlexikon, hg. von Kurt RuH u.a., Bd.6, Berlin/New York 21987, Sp. 1016-1035; auch: Andreas Deutsch, Die „Rethorica und Formulare teütsch“ des Pforzheimer Stadtschreibers Alexander Hugen - ein juristischer Bestseller des 16. Jahrhunderts, in: Neue Beiträge zur Pforzheimer Stadtgeschichte 2 (2008) S.31-75.

${ }^{42}$ Vgl. Andreas Deutsch, Der Klagspiegel und sein Autor Conrad Heyden - Ein Rechtsbuch des 15. Jahrhunderts als Wegbereiter der Rezeption, Köln/Weimar/Wien 2004, S.132f., 144f., 210; da Heyden nur bis 1436 amtierte, dürfte er als Ausbilder von Straub kaum in Betracht kommen, aber auch Heydens Amtsnachfolger hatten Schreibschüler. $\mathrm{Zu}$ Schreibschulen allgemein: Peter-Johannes Schuler, Geschichte des südwestdeutschen Notariats - von seinen Anfängen bis zur Reichsnotariatsordnung von 1512, Bühl (Baden) 1976, S. $102 \mathrm{f}$. 
lichen Notar, zum „notarius publicus apostolica auctoritate“, die freilich - aufgrund der eher rigiden Handhabung durch die päpstliche Kurie, eher selten war, oder die - wesentlich verbreitetere - Ernennung zum kaiserlichen Notar. Hierfür waren - in Vertretung des Kaisers - die sog. Hofpfalzgrafen (Comites palatini) zuständig ${ }^{43}$. Da sich Straub in zahlreichen Urkunden selbst als kaiserlicher Notar ${ }^{44}$, kaiserlicher Offenschreiber ${ }^{45} \mathrm{bzw}$. auf Lateinisch publicus sacra imperiali anctoritati notarius ${ }^{46}$ bezeichnet, ist klar, welchen Weg er gewählt hat.

Sicherlich wird der ihn ernennende Hofpfalzgraf Straubs hinreichende Bildung im Blick gehabt haben. Jedenfalls musste er darauf achten, dass der Kandidat von freier, ehrlicher und ehelicher Herkunft sowie unbescholten war - dies dürften vor Einführung der Reichsnotariatsordnung 1512 die einzigen festen Kriterien für die Kandidatenauswahl gewesen $\operatorname{sein}^{47}$. Daneben dürfte der Hofpfalzgraf auch schon im 15. Jahrhundert auf einer angemessenen Remuneration bestanden haben, in späteren Jahrhunderten wurden die Einnahmen aus der Titel- und Amtsvergabe zum regelrechten Erwerbsgeschäft für die zur Vornahme von Ernennungen Berechtigten ${ }^{48}$.

Bislang ließ sich nicht klären, welcher Hofpfalzgraf Straub ernannt haben könnte ${ }^{49}$. In der Literatur ist immer wieder zu lesen, dass auch Universitäten das Hofpfalzgrafenamt innehatten ${ }^{50}$. Die Universität Heidelberg erhielt dieses Privileg allerdings erst im Jahre $1745^{51}$, scheidet somit als mögliche Verleiherin des Notari-

43 Vgl. etwa Schmidt-Thomé (wie Anm. 17) insbesondere S. 265; Jürgen Arndt, Zur Entwicklung des kaiserlichen Hofpfalzgrafenamtes von 1355-1806, in: DERs. (Bearb.), Hofpfalzgrafen-Register, hg. vom Herold, Verein für Heraldik, Genealogie und verwandte Wissenschaften zu Berlin, Bd.1, Neustadt an der Aisch 1964, S.V-XXIV; DERs., Das Notarernennungsrecht der kaiserlichen Hofpfalzgrafen, in: Tradition und Gegenwart - Festschrift zum 175jährigen Bestehen eines badischen Notarstandes, hg. von Peter-Johannes SCHULER, Karlsruhe 1981, S.110-123.

${ }^{44}$ U 2370, in: UB Schwäbisch Hall (wie Anm. 26). Vgl. auch U 477, in: Vellberg in Geschichte und Gegenwart: Materialien, hg. von Hansmartin Decker-Hauff, Sigmaringen 1994.

${ }^{45}$ U 2453, in: UB Schwäbisch Hall (wie Anm. 26).

46 U 820, in: UB Heilbronn (wie Anm. 20).

47 Schmidt-Thomé (wie Anm. 17) S. 263.

48 Ebd., S. $266 \mathrm{ff}$.

${ }^{49}$ Keine Aufschlüsse geben die drei Bände: Jürgen Arndt (Bearb.), HofpfalzgrafenRegister, hg. vom Herold, Verein für Heraldik, Genealogie und verwandte Wissenschaften zu Berlin, Neustadt an der Aisch 1964-1988, da dort nur spätere Hofpfalzgrafen aufgenommen sind.

50 Etwa Hans-Wolf Thüмmel, Die Tübinger Universitätsverfassung im Zeitalter des Absolutismus, Tübingen 1975, S. 134.

51 Offiziell geschah dies in „Erneuerung des aus habender Spur der Universität erteilt gewesener Diplomae (!) Comitivae Palatinae, das durch Krieg und Brand entkommen“ sei, jedoch ist vor 1745 von einem Palatinat für die Universität Heidelberg keine Spur vorhanden, vgl. Erwin Sснміdт, Die Hofpfalzgrafenwürde an der hessen-darmstädtischen Universität Marburg/Gießen, Gießen 1973, S. 41. Das erste bekannte Privileg für eine Universität erhielt 
ats an Straub aus. Schon früh besaßen aber einige Leiter von Kanzleischulen die Ermächtigung zur Notars-Ernennung; so ist eher zufällig überliefert, dass Stadtschreiber Nikolaus von Wyle sacri Lateranensis pallacii anleque et consistorii imperialis comes war $^{52}$. Möglicherweise hat Straub den Notarstitel also am Ende seiner Ausbildung in einer solchen Schule erhalten. Denkbar erscheint ferner, dass sich Straub an den in unmittelbarer Nachbarschaft zu Heidelberg residierenden Speyerer Bischof gewandt hat, dessen Diözese er ja angehörte: Bereits Kaiser Karl IV. hatte 1355 dem damaligen Speyerer Bischof Gerhard das Amt eines Comes sacri Lateranensis palatii verliehen. Entsprechend der kaiserlichen Anordnung ging diese besondere Form des Hofpfalzgrafenamts auf Gerhards Amtsnachfolger über und war noch im 16. Jahrhundert in Gebrauch, wie einige aus dieser Zeit überlieferte Notarsernennungen durch den Speyerer Bischof belegen ${ }^{53}$. Leider ist nicht bekannt, welche Notare von den Fürstbischöfen des 15. Jahrhunderts ernannt wurden.

Ein am 20. Juli 1445 wohl in Heilbronn angefertigtes Vidimus über ältere Urkunden, unterzeichnet von Notar Nikolaus Strub aus Leonberg ${ }^{54}$, lässt vermuten, dass Straub weiterhin im Raum Heilbronn als Notar tätig war. Im Jahr darauf findet sich Straub in neuer Funktion, nämlich als Schultheiß zu Wimpfen im Tal. In diesem Amt übernahm er die Prozessvertretung für Eberhard von Finsterlohe vor dem geistlichen Gericht in Würzburg und wandte sich in diesem Zusammenhang am 1. Oktober 1446 in einem Schreiben an die Stadt Heilbronn ${ }^{55}$.

Leider liegt die Wimpfener Zeit Straubs völlig im Dunkeln ${ }^{56}$; die auf mehrere Archive verteilten Quellenbestände sind weiten Teils unerschlossen, sodass eine gezielte Suche nach bestimmten Akten derzeit unmöglich ist ${ }^{57}$. Erst 1457 stoßen

Helmstedt 1575, die Forschung geht davon aus, dass Heidelberg keinesfalls früher privilegiert worden sein kann, vgl. Felicitas RitTER, Universität Heidelberg - Prokanzler und Dekan der juristischen Fakultät 1745-1806, in: ARNDT, Hofpfalzgrafen-Register (wie Anm. 43) S. 155-163, hier S. 159.

${ }^{52}$ Reg.-Nr.12864 (Urkunde vom 30. Juni 1464, Stadtarchiv Überlingen), in: Regesta episcoporum Constantiensium, bearb. von Karl RiEDER, hg. von der Badischen Historischen Commission, Bd.4: 1436-1474, Innsbruck 1941. Hierzu auch Schuler, Geschichte des südwestdeutschen Notariats (wie Anm.42) S.126 und $106 \mathrm{f}$.

${ }^{53}$ Hierzu: Edward Beck, Über das Hofpfalzgrafenamt der Fürstbischöfe zu Speyer und Notarsbestallungen im 16. Jahrhundert, in: Der Deutsche Herold 47 (1916) S. 112-114.

${ }^{54}$ UB Heilbronn (wie Anm. 20) U 635.

55 Ebd., U 649.

56 In Wimpfen selbst ist Straub unbekannt, im dortigen Stadtarchiv findet sich nichts. Für die Unterstützung bei der Recherche sei dem dortigen Archivar, Herrn Haberhauer, gedankt.

${ }^{57}$ Nach Verlust der Reichsunmittelbarkeit durch den Reichsdeputationshauptschluss 1803 fiel die Stadt Wimpfen zunächst an Baden, das Ritterstift Wimpfen im Tal hingegen an Hessen-Darmstadt. Baden und Hessen stritten um die Souveränitätsrechte des Stifts, Baden stimmte schließlich einem Gebietstausch zu und trat Wimpfen an Hessen ab. 1805/1806 
wir wieder auf Straubs Spur. Vermutlich war er schon seit einiger Zeit als wandernder Notar unterwegs; jetzt ist er in Feuchtwangen: In einer Streitsache zwischen Schwäbisch Hall und Würzburg stellte er am 2. Februar 1457 eine Urkunde aus ${ }^{58}$. Vielleicht entstanden aus diesem Anlass die Kontakte zur Reichsstadt Hall; vielleicht erfuhr er, dass dort ein ortsansässiger Notar gebraucht werden konnte?

\section{Straubs Bibelübersetzung in Schwäbisch Hall}

Wenig später war Straub jedenfalls in der damals aufblühenden Salz- und Handelsstadt: Am 8. Januar 1459 trat er als Notar für das vor den Toren Schwäbisch Halls liegende Kloster Comburg in Erscheinung in einem Rechtsstreit wegen eines Weinbergs in Gelbingen, einem zur Reichsstadt Hall gehörigen Dorf ${ }^{59}$. Mehrfach wird Straub nun für die Abtei aktiv ${ }^{60}$. Zudem ist er für das nahe gelegene Kloster Schöntal tätig61.

Aber auch sonst häufen sich - wohl aufgrund der guten Schwäbisch Haller Archivüberlieferung - die erhaltenen von Straub ausgestellten Urkunden derart, dass es sinnlos wäre, auf alle einzugehen ${ }^{62}$. Straub war zwar als freier Notar in Schwäbisch Hall tätig - also keineswegs in reichsstädtischen Diensten, nahm aber insoweit eine beinahe offizielle Funktion ein, als er damals der einzige Notar in der Stadt war und mit seiner Tätigkeit den Stadtschreiber und die reichsstädtische Kanzlei - die ebenfalls zur Ausstellung von Urkunden berechtigt waren - entlastete. Es braucht also nicht zu verwundern, wenn der „kaiserliche Notar“ Straub

wurde das Umland Wimpfens durch Baden und Württemberg annektiert, und Wimpfen lag fortan als hessische Exklave zwischen diesen Ländern. Dabei blieb es bis nach dem Zweiten Weltkrieg, wo nun die beiden Länder Groß-Hessen und Württemberg-Baden um Wimpfen stritten, bis das Oberlandesgericht Stuttgart 1951 entschied, dass Wimpfen staatsrechtlich ein Teil Württemberg-Badens sei. Aufgrund dieses Hinundhers sind die Wimpfener Bestände bis heute zerstreut und in weiten Teilen unerschlossen. Für freundliche Auskünfte sei Herrn Prof. Dr. Friedrich Battenberg und Dr. Lars Adler vom Hessischen Staatsarchiv Darmstadt (Schreiben vom 16. August 2010) sowie Herrn Dr. Raimund J. Weber und Frau Dorothea Bader vom Landesarchiv Baden-Württemberg (HStAS/StAL; Schreiben vom 11. Juni und 16. August 2010) herzlich gedankt.

58 U 2312, in: UB Schwäbisch Hall (wie Anm. 26).

59 Landesarchiv Baden-Württemberg, StAL B 375 S U 470.

60 Abt von Murrhardt: Vidimus für Comburg (diverse Urkunden) „durch Notar Nikolaus Straub von Leuberg", Landesarchiv Baden-Württemberg, StAL B 375 S U 452, 17. Sept. 1459. Vgl. auch U 2450 und U 2452 in: UB Schwäbisch Hall (wie Anm. 26) beide von 1463.

61 B 503 I U 450, 21. Februar 1463 - ausgestellt in Hall für Schöntal.

62 4. Dezember 1459: U 2372, in: UB Schwäbisch Hall (wie Anm. 26) U 796, in: Regesten der Urkunden des Hospitals zum Heiligen Geist in der Reichsstadt Hall bis 1480, bearb. von Kuno UlshöFeR/Herta BeutTER (Forschungen aus Württembergisch Franken, Bd. 24), Sigmaringen 1998 (nachfolgend UB Hospital); 1461: U 2395 und U 2396, in: UB Schwäbisch Hall (wie Anm. 26); 1462: U 2434, in: ebd.; 1463: U 2450 und U 2452, in: ebd. 
auch in städtischen Amtsgebäuden, etwa im Rathaus ${ }^{63}$ oder in der Kinderschule ${ }^{64}$, Urkunden ausfertigte.

Die letzte erhaltene Urkunde, die Nicolaus Straub von Lenberg, Spirer Bistums, kaiserlicher Offenschreiber in Schwäbisch Hall ausstellte, ist ein Schenkungsvertrag über eine Hufe im zur Reichsstadt gehörigen Dorf Rieden und ein Haalhaus (Salinengebäude) als Unterpfand, aufgesetzt am 1. März $1463^{65}$. Ab Juli 1463 begegnen dann andere Namen als kaiserliche Notare in Schwäbisch Hall: Zweimal ein Seifried Weierman aus Dinkelsbühl, Kleriker der Augsburger Diözese und kaiserlicher Notar ${ }^{66}$; dann im März 1464 ein Johannes Baldung, Kleriker der Augsburger Diözese und kaiserlicher Notar ${ }^{67}$ und ab 1465 ein Notar Sieder, der sich wie zuvor Straub - als Haller Notar bezeichnete ${ }^{68}$.

Da Nikolaus Straub sich am Ende seiner Übersetzung der vier Evangelien selbst als notarius hallensis bezeichnet, muss das Werk in Schwäbisch Hall entstanden sein. Mehr noch: Da Straub nur wenige Jahre - nämlich nur zwischen (frühestens) 1457 und (spätestens) 1465 in Schwäbisch Hall tätig war, vermutlich sogar nur zwischen 1458/1459 und 1463, lässt sich die Bibelübersetzung unerwartet genau datieren. Tendenziell lassen sich die benannten fünf bis sechs Jahre noch dahingehend einschränken, dass die Entstehung der Übersetzung eher gegen Ende von Straubs Schwäbisch Haller Zeit liegen dürfte, denn mutmaßlich wird er sich erst dann als notarius hallensis bezeichnet haben, als er sich in der Reichsstadt in diesem Amt etabliert hatte. Möglicherweise sollte ihm die Übersetzung dazu dienen, sich für eine neue Beschäftigung anzudienen - gewissermaßen als Beleg für umfangreiche Lateinkenntnisse und die Befähigung, längere Texte aufzusetzen.

\section{Aufstieg zum Generalsyndikus von Heilbronn}

1465 war Straub wieder in Heilbronn ${ }^{69}$. Er scheint dort zu dieser Zeit bereits eine bekannte und etablierte Persönlichkeit gewesen zu sein, denn er übernahm eine entscheidende Rolle bei der Reformation zweier Heilbronner Klöster: Der Maulbronner Abt Johann IV. von Wimsheim (1462 bis 1467) hatte von Papst Paul II. am 5. Oktober 1465 den Auftrag erhalten, gemeinsam mit seinem Amtskollegen Bernhard von Hirsau zwei Heilbronner Klöster zu reformieren; sowohl gegen die

${ }^{63}$ Urkunde vom 27. Oktober 1459: U 2368, in: UB Schwäbisch Hall (wie Anm.26); U 793, in: UB Hospital (wie Anm. 62).

${ }^{64}$ U 2370, in: UB Schwäbisch Hall (wie Anm. 26). Vgl. auch U 477, in: Decker-Hauff (wie Anm.44) 13. November 1459.

${ }^{65}$ U 2453, in: UB Schwäbisch Hall (wie Anm. 26).

${ }^{66}$ Urkunden vom 8. Juli 1463 und 22. Oktober 1464, in: ebd., U 2457 und U 2476.

67 Urkunde vom 27. März 1464, in: ebd., U 2467.

68 Urkunde vom 29. Mai 1465, in: ebd., U 2491; dann öfter.

${ }^{69} \mathrm{Vgl}$. auch die beiden von Straub ausgestellten Urkunden von 1465 und 1467, die in den Einband des unten beschriebenen Institutionenkommentars eingebunden sind. 
Heilbronner Franziskaner als auch gegen die dortigen Klarissen bestand der Vorwurf, sich nicht mehr hinreichend an die jeweiligen Ordensregeln - insbesondere das Armutsgelübde - zu halten ${ }^{70}$. Am 2. Dezember sollte die Reform beginnen, zunächst bei den „Barfüßern“: Mit mehreren Zeugen, unter anderem den Bürgermeistern von Heilbronn, begaben sich die beiden Äbte zum Kloster - als Rechtskundigen zogen sie Nikolaus Straub bei. Die Mönche verweigerten der Kommission jedoch zunächst den Einlass, erst nachdem die Reformer die Anwendung von Gewalt androhten, wurde ihnen die Pforte geöffnet. Nur widerwillig versammelten sich die Brüder im Kapitelsaal, wo Straub die päpstliche Bulle verlas. Die Mönche beteuerten ihre Unschuld und verweigerten jede Kooperation. Der Guardian und Lektor der Franziskaner Eberhard Sartoris bezeichnete die päpstliche Bulle als aufgrund unrichtiger Erzählungen erschlichen und kündigte eine Appellation an den Papst an. Aufgrund des unerlaubten Eindringens ins Kloster sei die gesamte Kommission im päpstlichen Bann. Hiervon ließen sich die Äbte freilich nicht beeindrucken.

Im Zentrum der Vorwürfe stand die Nichteinhaltung des Armutsgelübdes; man ließ dieses daher - zusammen mit anderen Ordensregeln - verlesen, doch die Mehrzahl der Mönche zeigte sich uneinsichtig. Daher besichtigte man die Habseligkeiten der Mönche, die ja nicht umsonst „Barfüßer“ genannt wurden, sollten sie doch außer einfachster Kleidung und Sandalen nichts besitzen. Man fand Kleider, Wamse, Schuhe, ja sogar Stiefel; außerdem hatten die Mönche Betten. Als erste Maßnahme unterstellte die Kommission die Mönche der Oberaufsicht eines bereits reformierten Klosters; der Guardian und Lektor wurde abgesetzt und exkommuniziert. Vom 6. Dezember 1465 datiert ein an den Papst gerichteter umfassender Bericht über die Durchsuchungen im Kloster - unterzeichnet: Et ego Nicolaus Straub de Lemberg Spirensis dyocesis publicus sacra imperiali auctoritati notarius etc. ${ }^{71}$ Durch dieses Protokoll sind die Abläufe überliefert.

Die Barfüßer, insbesondere der exkommunizierte Guardian und Lektor Sartoris, gaben nachvollziehbarerweise nicht klein bei, sondern sträubten sich so gut sie konnten gegen die Reformation. Am 9. Dezember erhob Sartoris im Schöntaler Hof zu Heilbronn formell Appellation gegen die vorgeblichen Reformatoren, die bewaffnet in das Kloster eingedrungen seien. Zwei davon hätten sich als Äbte ausgegeben, hätten keine Originalbulle des Papstes vorgewiesen, hätten auch nichts gefunden, was gegen die Ordensregeln verstoßen hätte. Auch vor Heinrich Furrer, dem Dekan von Stift Haug bei Würzburg ${ }^{72}$, klagte Sartoris; dieser jedoch war nicht

70 Karl Klunzinger, Urkundliche Geschichte der vormaligen Cisterzienser-Abtei Maulbronn, Stuttgart 1854, S. 121.

71 UB Heilbronn (wie Anm. 20) U 820.

72 Im Regest steht „Heinrich Fuirer“ als „Decan von St. Johann außerhalb der Mauern Heilbronns“, gemeint sein dürfte aber Würzburg, denn bei Heilbronn gibt es kein Dekanat St. Johann. Stift Haug ist eigentlich Stift St. Johann in Haug. 
nur Lizenziat des kanonischen Rechts und apostolischer Delegat, sondern zählte auch zu den bedeutenden Unterstützern der Reformbewegung. Er erklärte im Beisein der beiden Äbte und von Nikolaus Straub die Appellation an den Papst für formell und materiell ungültig. Sartoris bleibe exkommuniziert. Am 21. Dezember wiederholte Sartoris seine Klage in Würzburg, schlug sie an der Türe des Doms an; offenbar suchte er nun überregional nach Verbündeten.

In dieser Situation sahen auch die Heilbronner Handlungsbedarf. Der städtische Magistrat erwog, einen Gesandten zum Papst zu schicken, um dort Bericht zu erstatten - und Schlimmeres zu verhindern ${ }^{73}$. Abt Johann von Maulbronn riet den Heilbronnern daraufhin in einem Brief vom 21. Januar 1466, Nikolaus Straub nach Rom zu entsenden, weil Straub der Ding Fliß, ernst und erfarung habe ${ }^{74}$, sich besser auskenne als irgend ein anderer ${ }^{75}$.

Viel lässt sich über diese Romreise nicht in Erfahrung bringen. Am 3. Juni 1466 wandte sich Abt Eberhard vom Mönchberg - es dürfte sich um den bedeutenden Reformabt Eberhard III. vom St. Michaelsberg in Bamberg handeln - an die Stadt Heilbronn mit der Bitte, man möge ihm die zweiundzwanzig Dukaten, die er in dieser Sache geliehen habe, nach Nürnberg oder Bamberg übersenden ${ }^{76}$. Dies könnte dafür sprechen, dass Straub zu diesem Zeitpunkt bereits wieder aus Rom zurück war. Er begegnet nun in verschiedenen Urkunden als Notar Mag. Nikolaus Straub, erwirkte beispielsweise am 30. August 1468 als Prokurator oder Syndikus für Schöntal ein Vidimus von verschiedenen Urkunden durch den Propst von Wimpfen im Tal7 ${ }^{7}$. Straub ist ferner als Heilbronner Anwalt in Heidelberg aktiv ${ }^{78}$.

In der Landesbibliothek Speyer steht als Buch Nr. 1 - die älteste Handschrift aus dem Altbestand der Bibliothek - ein über 600 Seiten starkes Manuskript, auf das ich nur durch große Zufälle aufmerksam geworden bin ${ }^{79}$. Es handelt es sich um ein weiteres Buch des Nikolaus Straub; ganz ähnlich wie die Evangelien-Überset-

73 Klunzinger (wie Anm. 70) S. 121.

${ }^{74}$ Carl JäGER, Mittheilungen zur schwäbischen und fränkischen Reformationsgeschichte, nach handschriftlichen Quellen, Bd.1, Stuttgart 1828, S.17.

${ }^{75}$ UB Heilbronn (wie Anm.20) U 820e. Das Regest zu dieser Urkunde missversteht Schuler, weshalb dieser fälschlich annimmt, Straub sei Klosterpfleger bzw. Fabrikmeister für ein Heilbronner Kloster gewesen, was dieser aller Wahrscheinlichkeit nie war, denn in diesem Amt werden in den Quellen immer wieder andere Personen genannt (s. etwa UB Heilbronn (wie Anm. 20) U 1535b, i, k); vgl. Schuler, Notare Südwestdeutschlands (wie Anm. 22) Nr.1326, S.450, dem irrtümlich folgend der Verfasser, Der Klagspiegel und sein Autor Conrad Heyden (wie Anm. 42) S. 109.

76 Der 1463 vom Papst zur Reform des Klosters eingesetzte Abt stammt aus Venlo, was die teils niederdeutsche Schreibweise in der Urkunde erklärt; vgl. Johann Samuel Ersch/ Johann Gottfried Gruber, Allgemeine Encyclopädie der Wissenschaften und Künste, Erste Section, 30. Theil, Leipzig 1838, S. 32.

77 Landesarchiv Baden-Württemberg, StAL B 503 I U 218.

78 UB Heilbronn (wie Anm. 20) U 874, 6. April 1470.

79 Vgl. den unspezifischen Hinweis bei:Jürgen Vorderstemann, Die Büchersammlungen des Speyerer Domes in tausend Jahren, in: Archiv für Mittelrheinische Kirchengeschichte 33 
zung hat es ein kurzes Nachwort mit Straubs Unterschrift, beides ist diesmal allerdings - wie das gesamte Werk - lateinisch:

Finito que libro reddatur gratia Christo ${ }^{80}$ [nun andere Farbe] anno domini milesimo quadrigentesimo septuagesimo primo, die Jouio [Jovis = Donnerstag] ultimo Januarii ${ }^{81}$ Regnantibus In spiritalibus paulo papa $2^{\circ}$ anno eius septimo ${ }^{82}$ In temporalibus autem Invictissimo Friderico romanorum Imperatore me nicolao straub In opido beylpronno tunc sindico generali Institutus.

Quo tempore totus mundus In oppinione erat et tribulationem propter regentium tyrannidem ${ }^{83}$.

Bei dem Werk handelt es sich um einen Kommentar zum römischen Recht, genauer: zu den Institutionen aus dem Corpus Iuris Iustiniani, einer knappen, lehrbuchartigen Zusammenfassung der gesamten justinianischen Gesetzgebung. Der große Umfang lässt vermuten, dass Straub an dem Werk etliche Monate, wenn nicht Jahre gesessen hat, bevor er es am 31. Januar 1471 abschloss. Grundlage könnten Materialien sein, die er von seiner Romreise mitgebracht hat. Einzelne teils lateinische, teils deutschsprachige Randbemerkungen wie quid ius naturale oder zoberey sollten den Zugriff auf den - aufgrund zahlloser Abbreviationen nur schwer lesbaren - lateinischen Text erleichtern, den zu erforschen der Rahmen dieses Beitrags nicht zulässt.

Für Straubs Biographie ist der zitierte Schlusssatz von besonderer Bedeutung, da er darin hervorhebt, dass er nunmehr als Generalsyndikus der Stadt Heilbronn eingesetzt sei. Es scheint, als sei das Amt des Stadtsyndikus speziell für Nikolaus Straub neu geschaffen worden, sodass mit dem Datum 1471 auch ein Einschnitt in der Heilbronner Verfassungsgeschichte festgehalten werden kann. Carl Jäger, der noch auf Quellen zurückgreifen konnte, die im Zweiten Weltkrieg zerstört wurden, berichtet, Straub habe als städtischer Syndikus unter anderem bei Gericht sitzen, bei der Findung von Urteilen helfen und diese aufschreiben müssen ${ }^{84}$.

(1981) S. 45-61, 56f. S. auch: Catalog der Lyceumsbibliothek zu Speyer, Speyer 1828, S.3, Nr.1.

${ }_{80}$ Diese im Kern häufige Formel findet sich mit dem selteneren „Finitoque“ beispielsweise auch am Ende einer Ausgabe des Decretum Gratiani vom Ende des 13. Jahrhunderts, vgl. Zéphir François Cicéron Caron, Catalogue des manuscrits de la bibliothèque de la ville d'Arras, Arras 1860, S. 684 (zu Nr. 577).

81 Der 31. Januar 1471 war tatsächlich ein Donnerstag.

82 Paul II. wurde am 30. August 1464 zum Papst gewählt und starb am 26. Juli 1471.

${ }^{83}$ Dies ist wohl eine Anspielung auf den Konflikt zwischen Kaiser Friedrich III. und dem einflussreichen Kurfürsten Friedrich dem Siegreichen von der Pfalz, der selbst Machtbestrebungen über das Reich hatte; kriegerische Auseinandersetzungen zwischen Pfalz und Württemberg hatten auch die Region um Heilbronn betroffen.

${ }^{84}$ Für Jäger scheint Straub allerdings erst 1480 nachweisbar zu sein; vgl. Carl JäGER, Geschichte der Stadt Heilbronn und ihres ehemaligen Gebietes - ein Beitrag zur Geschichte des schwäbischen Städtewesens, Bd.1, Heilbronn 1828, S.251. In der Fußnote bezieht sich Jäger allerdings auf ein Gerichtsprotokoll von 1490. 
Von zentraler Bedeutung waren aber neben der Rechtsberatung des Magistrats vor allem diplomatische Missionen.

\section{Zweite Romreise und die Affäre Cock}

Nur indirekt erfahren wir von einer zweiten Romreise Straubs: In einer Urkunde vom 4. Januar 1472 wird erwähnt, dass Straub der von Wimpfen wegen in Rom gewesen sei $^{85}$. Vermutlich stand auch diese Reise mit der Reform einer geistlichen Einrichtung im Zusammenhang: Im Jahr 1471 erwirkte die Stadt Wimpfen nämlich die Erlaubnis des Präzeptors des Heilig-Geist-Ordens ${ }^{86}$ in Rom zur Trennung des weltlichen Siechenhauses vom geistlichen „Hospital zum Heiligen Geist“. Diese beiden Sozialeinrichtungen waren 1421 unter geistlicher Führung vereinigt worden, doch gänzlich ohne den erhofften Synergieeffekt; wachsende Probleme machten die Rückgängigmachung der Fusion unumgänglich. Von der Teilung dürfte nun vor allem die Stadt profitiert haben, auch wenn sie offiziell nicht zuletzt den Conventsbrüdern zu einer bequemen müssigen Rube geschah ${ }^{87}$. Die Teilung erfolgte 1471 am Tag Francisci, also am 3. Oktober ${ }^{88}$, ob Straub zu diesem Zeitpunkt wieder aus Rom zurück war, ist offen.

Am 3. September 1471 begann eine der erbittertsten Streitigkeiten, in welche Straub während seiner beruflichen Laufbahn verwickelt war: Nach dem Tod des Heilbronner Pfarrers Johann Gemminger setzte Papst Sixtus IV. den (streitgeübten ${ }^{89}$ ) Kanoniker Doktor Albert Cock in die Heilbronner Pfarrei ein ${ }^{90}$. Der aus Norddeutschland stammende und in Erfurt promovierte Kirchenrechtler gehörte

85 UB Heilbronn (wie Anm. 20) U 893 e.

${ }^{86} \mathrm{Zu}$ den Strukturen dieses Ordens vgl. Gisela Drossbach, Christliche caritas als Rechtsinstitut. Hospital und Orden von Santo Spirito in Sassia (1198-1378), Paderborn 2005; Andreas Rehberg, Päpstliche Caritas auf dem Prüfstand - Anmerkungen zu einer neuen Studie zum Heilig-Geist-Orden, in: Quellen und Forschungen aus italienischen Archiven und Bibliotheken 86 (2006) S. 562-581.

87 Vgl. Ludwig Frohnhäuser, Geschichte der Reichsstadt Wimpfen, des Ritterstifts St. Peter zu Wimpfen im Thal, des Dominicanerklosters und des Hospitals zum hl. Geist zu Wimpfen am Berg, Darmstadt 1870, S. $149 \mathrm{ff}$.

88 Die Urkunde findet sich in: Johann Jacob Moser, Reichs-Stättisches Hand-Buch, worinn die heutiges Tages noch einen Nutzen zu haben scheinende Urkunden, welche des Heiligen Römischen Reichs Frey- und Reichs-Stätte überhaupt oder insbesondere angehen [...] anzutreffen seynd, Bd.2, Tübingen 1733, S.914f.

89 Vgl. etwa: Adolf Gоттlob, Aus der Camera Apostolica des 15. Jahrhunderts, ein Beitrag zur Geschichte des päpstlichen Finanzwesens, Innsbruck 1889, S. 134.

90 Sehr knapp hierzu auch: Gertrud RückLIN, Religiöses Volksleben des ausgehenden Mittelalters in den Reichsstädten Hall und Heilbronn (Historische Studien, Bd. 226), Berlin 1933, S. 44 . 
spätestens ${ }^{91}$ seit 1457 der päpstlichen Kanzlei als Abbreviator an ${ }^{92}$, war also einer jener hochrangigen Beamten, welche für die formkorrekte Ausformulierung offizieller Schriftstücke im Umkreis der Kurie - etwa auch der Eingaben an den Papst-zuständig waren ${ }^{93}$. Im Aufsetzen von Suppliken geübt ${ }^{94}$, hatte Cock (wie die meisten seiner Kollegen) bereits mehrere Bittschriften eingereicht, um vom Papst als Gegenleistung für treue Dienste - mit Pfründen im Reich, vor allem in Norddeutschland, ausgestattet zu werden ${ }^{95}$. So wurde Cock beispielsweise 1467 mit der bedeutenden Propstei von Bardowick (bei Lüneburg) providiert ${ }^{96}$. Später bemühte er sich vergeblich um die Propstei der Stephanskirche in Konstanz ${ }^{97}$; ab Dezember 1478 ist der decret. doctor et literarum apostolicarum abbreviator dann aber als Dompropst in Bremen nachweisbar, wo er schließlich im Herbst 1485 verstarb ${ }^{98}$. Cocks Bemühungen um die Heilbronner Pfarrei waren somit für ihn eher Routine. Als sich Cock (ohne Rom zu verlassen) mit Urkunde vom 14. September 1471 in Bezug auf die Heilbronner Pfarrei verpflichtete, hob er die dortigen (im Vergleich zu seinen zuvor erworbenen Pfründen stattlichen) Einnahmen hervor, die auf im-

${ }^{91}$ Cock erscheint bereits in einer Supplik vom 24. Oktober 1454, vgl. Repertorium Germanicum - Verzeichnis der in den Registern und Kameralakten vorkommenden Personen, Kirchen und Orte des Deutschen Reiches, seiner Diözesen und Territorien (RG), hg. vom Deutschen Historischen Institut in Rom, Berlin u. a. 1897 ff.; Bd. VI, Nr. 00072.

${ }_{92}$ Ulrich Schwarz, Petenten, Pfründen und die Kurie: Norddeutsche Beispiele aus dem Repertorium Germanicum, in: Blätter für deutsche Landesgeschichte 133 (1997) S.1-21, hier S. 9.

${ }_{93}$ Zur Aufgabe der Abbreviatoren: Walther von Hofmann, Forschungen zur Geschichte der kurialen Behörden vom Schisma bis zur Reformation, Bd.1, Rom 1914, S. $120 \mathrm{ff}$.

94 Vgl. nur die Einträge im Repertorium Germanicum (wie Anm. 91) zu Albertus Cock, RG, Bd.VI, Nr. 00072; Bd. VII, Nr.00043; Bd. VII, Nr. 02020; Bd. VII, Nr. 02702; Bd. VIII, Nr.00080; Bd.VIII, Nr. 01887; Bd.VIII, Nr.03491; Bd.VIII, Nr. 04807; Bd.IX, Nr.00066; Bd.IX, Nr. 00394; Bd. IX, Nr. 00547; Bd. IX, Nr.00710; Bd. IX, Nr. 00918; Bd. IX, Nr.01103; Bd.IX, Nr. 01198; Bd.IX, Nr. 01256; Bd.IX, Nr. 01276; Bd. IX, Nr. 01370; Bd. IX, Nr. 01636; Bd.IX, Nr.01885; Bd. IX, Nr. 01906; Bd.IX, Nr. 02110; Bd. IX, Nr. 02191; Bd. IX, Nr. 02246; Bd.IX, Nr. 02416; Bd. IX, Nr. 02561; Bd. IX, Nr. 02738; Bd. IX, Nr. 02860; Bd. IX, Nr. 03188; Bd.IX, Nr. 03340; Bd. IX, Nr. 03395; Bd. IX, Nr. 03590; Bd. IX, Nr. 03679; Bd. IX, Nr. 03680; Bd. IX, Nr. 04050; Bd. IX, Nr. 04033; Bd. IX, Nr. 04079; Bd. IX, Nr. 04298; Bd. IX, Nr. 04301; Bd.IX, Nr.05726; Bd. IX, Nr.06042; Bd.IX, Nr. 06096.

95 Schwarz (wie Anm. 92) S. 10, nennt einige davon.

${ }^{96}$ Hierzu auch eine Anmerkung in: Die Chroniken der fränkischen Städte - Nürnberg, Bd. 5 (Chroniken der deutschen Städte vom 14. bis ins 16. Jahrhundert, Bd.11), Leipzig 1874, S. $770 \mathrm{f}$.

97 Schwarz (wie Anm. 92) S. 10, mit weiteren Nachweisen.

98 „die Anthonii“ (wohl 2. September), vgl. Johann Melchior Kohlmann, Verzeichnisse der Dompröpste, Archidiakonen und sonstigen Dignitäten der Bremer Kirche, in: Wilhelm von Hodenberg, Die Diöcese Bremen und deren Gaue in Sachsen und Friesland, Bd.3 (Beilagen), Celle 1859, S.17-38, 21. 
merhin zehn Mark Silber geschätzt wurden - ein klarer Hinweis, worauf es ihm ankam $^{99}$.

Fürstbischof Rudolf II. von Würzburg hatte allerdings schon längst einen gewissen Johann Dunzenbach in die Pfarrei eingesetzt und dachte gar nicht daran, seinen Kandidaten fallen zu lassen; Dunzenbach wiederum hatte drei Geistliche als Vertreter berufen, um nicht selbst seelsorgerisch aktiv werden zu müssen. Damit schienen hinreichend Fakten geschaffen, um den vom Papst ernannten Cock abzuwehren. Mehrere Schreiben päpstlicher Delegierter ließen den Bischof daher kalt.

Albert Cock jedoch erwirkte am 21. November 1471 in Rom ein Mandat durch den speziell delegierten Auditor, den päpstlichen Kaplan und Erzpriester zu Bologna Dr. Antonius de Grassis, in dem dieser dem Bischof anordnete, binnen sechs Tagen nach Bekanntwerden des Mandats das Volk zur Vertreibung Dunzenbachs zu veranlassen, ihn zur Rückgabe seiner Einkünfte zu bewegen und Cock in die Pfarrei einzuweisen. Offenbar nutzte Cock dieses Mandat, um - den damals vielleicht noch in Rom weilenden, jedenfalls Cock durch seine Romfahrt persönlich bekannten - Straub mit der Sequestration zu betrauen. So drang Straub im Dezember des Jahres in die Kilianspfarrei ein und nahm die Kasse und allerlei Unterlagen an sich. Der Würzburger Bischof reagierte mit einem geharnischten Brief an die Heilbronner Stadtoberen ${ }^{100}$. Diese wiederum scheinen die Konfrontation mit dem Fürstbischof nicht gescheut zu haben. Offiziell wuschen sie die Hände in Unschuld, behaupteten in einem Schreiben vom 4. Januar 1472, die Stadt habe mit der Angelegenheit nichts zu tun, mische sich nicht in geistliche Dinge. Da sich Straub zu dieser Zeit aber bereits „Syndikus (bzw. Generalsyndikus) von Heilbronn“ nannte, ist es recht unwahrscheinlich, dass er ohne Zustimmung des Magistrats handelte. Der Heilbronn-Forscher Carl Jäger vermutet denn auch politische Ränkespiele der Reichsstadt ${ }^{101}$.

Schließlich erging am 20. Juli 1472 in Rom ein Erlass durch den päpstlichen Kaplan und Erzpriester zu Bologna Dr. Antonius de Grassis, in dem der „verwegene Eindringling“ Dunzenbach aus der Heilbronner Pfarrei förmlich „entfernt“ wurde. Er sei zudem verpflichtet, alle seine Einkünfte zurückzuerstatten und die Prozesskosten Cocks zu tragen, zudem wurde ihm der Klageweg abgeschnitten. Mit der Exekution des Erlasses wurde der Wormser Dekan Dr. Johann Enolfi betraut. Dieser wiederum bestellte mit Schreiben vom 24. August 1472 den Notar und Heilbronner Syndikus Nikolaus Straub nun formell zum Sequestrator. Straub sollte zudem die Führung der Seelsorge durch einen Vizepleban und die örtliche Geistlichkeit organisieren ${ }^{102}$.

991472 wurden die Einkünfte auf achtzehn Mark Silber präzisiert, UB Heilbronn (wie Anm. 20) U 122.

100 Ebd., U 893e.

101 JäGER, Mittheilungen zur schwäbischen und fränkischen Reformationsgeschichte (wie Anm. 74) S.3.

102 UB Heilbronn (wie Anm. 20) U 893g. 
Dass Dunzenbach nicht einfach aufgeben würde, verwundert wenig; in Anbetracht des Umstandes, dass der Dekan von St. Peter zu Wimpfen im Tal als Richter bestimmt wurde, ist aber ebenso klar, dass die Dinge für den einstigen Wimpfener Schultheißen Straub - und damit für seinen Mandanten Albert Cock sehr gut standen. Jäger sieht in der letztendlich erfolgreichen Vertreibung Dunzenbachs einen Reformerfolg der Heilbronner; immerhin war Cock u. a. durch den Reformer Abt Eberhard vom St. Michaelsberg in Bamberg unterstützt. Allerdings hatte Cock mit Heilbronn nicht viel am Hut, ist wahrscheinlich kein einziges Mal in der Neckarstadt gewesen. Nach seiner Zeit in Rom wurde Cock Propst von St. Peter und Paul in Bardowick, der berühmten Backsteinkirche bei Lüneburg. Dorthin mussten die Heilbronner nun die Einkünfte der Kilianskirche - als jährliches Leibgeding in Höhe von zehn Goldgulden - überweisen ${ }^{103}$.

Aus den folgenden Jahren existieren hinreichend Urkunden, um Straubs weitere Tätigkeit in Heilbronn zu belegen ${ }^{104}$. Wegen unterschiedlichster Rechtsangelegenheiten ist er auch als Notar oder Anwalt aktiv, so im Auftrag von Reichsstadt oder Bürgern wiederholt auf Reisen, unter anderem mehrfach in den benachbarten Residenzstädten Heidelberg ${ }^{105}$ und Stuttgart ${ }^{106}$.

Wahrscheinlich war Straub als Syndikus zudem an der Redaktion von reichsstädtischen Statuten und sonstigen Normtexten zumindest beteiligt. Erwähnenswert erscheint in diesem Zusammenhang die recht bekannte „Heilbronner Turnier-

103 Vgl. Urkunde vom 12. Juni 1476, ebd., U 893 q.

104 11. Oktober 1475: Vidimus von UB Heilbronn (wie Anm. 20) U 874 durch den Rektor der Heidelberger Universität (bzw. dessen Pedellen) für den Heilbronner Syndicus Nikolaus Straub; 6. Mai 1476: Vidimus von UB Heilbronn (wie Anm. 20) U 874 durch Straub für Bürgermeister von Heilbronn, ebd., U 874 a; 1476: in einer Würzburger Urkunde vom 10. März 1476 wird ein „instrumentum publicum“ über die Stiftung zweier Jahrmessen in der Heilbronner Pfarrkirche genannt, das Testament des dortigen Vikars Martin Steger hatte der öffentliche Notar „Nikolaus Strawbe“ aufgesetzt - wann wird nicht erwähnt, vgl. Urkundenregesten zur Geschichte der kirchlichen Verwaltung des Bistums Würzburg (1136-1488), bearb. von Wilhelm EngeL, Würzburg 1954, Nr.309, S. 237 f.; 13. November 1479: Straub als Fürsprech in Stuttgart, UB Heilbronn (wie Anm. 20) U 1165 h; 1479: als Anwalt Heilbronns in Heidelberg vor dem Pfalzgrafen/Hofgericht, ebd., U 1261 a.; 17. März 1480: Vidimus für Prior, ebd., U 1233; 10. Dezember 1481: Straub als Fürsprech für Böckinger Bürger vor dem Hofgericht in Stuttgart, ebd., U 1326; 13. April 1483: Als Heilbronner Syndikus überreicht Meister Niklaus Straub $50 \mathrm{fl}$. in Dilsberg im Auftrag einer Familie wegen einer Vormundschaftssache, ebd., U 1136; 1. Febr. 1485: Straub siegelt als Syndikus von Heilbronn eine Urkunde für den Pfarrer von Neckargartach (bei Heilbronn), ebd., U 1394.

105 12. Oktober 1475: Vidimus von UB Heilbronn (wie Anm. 20) U 874 durch den Rektor der Heidelberger Universität (bzw. dessen Pedellen) für den Heilbronner Syndikus Nikolaus Straub; 1479: als Anwalt Heilbronns in Heidelberg vorm Pfalzgrafen/Hofgericht, ebd., U 1261 a.

106 13. November 1479: Straub als Fürsprech in Stuttgart, ebd., U 1165 h; 10. Dezember 1481: Straub als Fürsprech für Böckinger Bürger vor dem Hofgericht in Stuttgart, ebd., U 1326. 
ordnung“ von 1485107. Die Repräsentanten der in den „Vier Landen“ (Franken, Schwaben, Rheinland und Bayern) zusammengeschlossenen ritterlichen Turniergesellschaften waren in diesem Jahr in Heilbronn zusammengekommen, um sich eine einheitliche Satzung über die Zulassung zum Turnier, dessen Ablauf und die Strafen bei Regelverstößen zu geben; im Zentrum stand die Verhinderung zu hoher Kosten und der Ausschluss nichtritterlicher (insbesondere stadtadeliger) Teilnehmer. Wer die Ordnung redigiert hat, ist leider nicht überliefert ${ }^{108}$.

\section{Dritte Romreise}

Im seit 1483 schwelenden Streit um die Reform des erst um die Mitte des 15. Jahrhunderts vor den Toren der Stadt Heilbronn errichteten Karmeliterklosters hatte der Würzburger Bischof im April 1485 einen Vergleich zwischen Mönchen und Reichsstadt erwirken können, der aber - ausweislich der bischöflichen Bulle - noch vom Papst genehmigt werden musste ${ }^{109}$. Der Vergleichsvertrag vom 4. August 1485 sah zudem eine Bestätigung durch die Ordensspitze in Rom vor ${ }^{110}$.

Wenige Tage darauf erteilten der Ordensprovinzial der Karmeliter Johannes Carpentarius (alias Zimmermann), der Heilbronner Prior und der Konvent Magister Nikolaus Straub in seiner Funktion als Generalsyndikus der Stadt Heilbronn die Vollmacht, vom Ordensgeneral Franciscus Pontius Rainaudus ${ }^{111}$ und anschließend vom Papst Bestätigungen des geschlossenen Vergleichs einzuholen. Unklar war allerdings zunächst, wo der Ordensgeneral anzutreffen sei. Aufgrund des Schismas kam neben Rom Avignon in Betracht; nach Informationen des Heilbronner Rats, sei der General aber nicht dort, sondern im irischen Auion; aus Karmeliterkreisen wurde daraufhin versichert, ihr Oberster sei in Rom zu finden. So wurde beschlossen, Straub solle ausschließlich dorthin ${ }^{112}$ reisen $^{113}$.

107 Abgedruckt z.B. in: Melchior Goldast (Hg.), Reichshandlung und andere deß H. Römischen Reichs Acta, Tractaten, Keyserliche, Königliche und Fürstliche Mandata, beyde Geistlich und Weltlich Regiment betreffendt, Hanau 1609, S. 25 ff.; Jean-Marie MoegLIN/ Rainer A. Müller (Hg.), Deutsche Geschichte in Quellen und Darstellung, Bd.2: Spätmittelalter 1250-1495, Stuttgart 2005, S.456ff.; vgl. zudem etwa Cord UlRichs, Vom Lehnhof zur Reichsritterschaft, Strukturen des fränkischen Niederadels am Übergang vom späten Mittelalter zur frühen Neuzeit, Stuttgart 1997, S. 135 ff. mit weiteren Nachweisen.

108 Vgl. auch UB Heilbronn (wie Anm. 20) U 1400 mit weiteren Nachweisen.

109 Vgl. ebd., U 1366 g.

110 Ebd., U $1366 \mathrm{k}$.

111 Der Name steht nicht in den Heilbronner Unterlagen, ergibt sich aber aus der Ordensgeschichte, vgl. etwa: Louis de SAInTe Thérèse, La succession du Saint prophète Elie en l'ordre des Carmes, Paris 1662, S.577; Carolus Vaghus de Parma, Commentaria fratrum et Sororum Ordinis B.M. V. de Monte Carmelo, Parma 1725, S. 60. Pontius Rainaudus wurde 1481 in Avignon zum Ordensgeneral gewählt.

112 Straub reiste demnach nicht nach Avignon über Basel, Solothurn, Bern und Genf, wie Schuler schreibt, vgl. Schuler, Notare Südwestdeutschlands (wie Anm. 22) Nr. 1326, S. 450. Hier liegt wohl ein Missverständnis von UB Heilbronn (wie Anm. 20) U 1366 n vor.

113 Ebd., U 13661-n. 
Bevor sich Straub auf die Reise machen konnte, mussten Empfehlungsschreiben beschafft werden, ohne die an der päpstlichen Kurie nichts zu erhoffen war ${ }^{114}$ : Ein Brief des Kurfürsten Philipp des Aufrichtigen von der Pfalz, einer des Grafen Eberhard von Württemberg, einer des Bischofs Rudolf II. von Würzburg und einer des Doktor Kilian von Bibra, des damaligen Dompropsts in Würzburg, einer in Kirchenkreisen allgemein anerkannten Persönlichkeit ${ }^{115}$.

Straubs Reisebericht enthält erstaunliche Details über seine nun folgenden Abenteuer. Über Rom lag zu dieser Zeit nämlich die Pest; wer es sich leisten konnte, hatte die Stadt längst verlassen. Glücklicherweise hatte Straub, als er durch Senis (also Siena) kam, erfahren, wo er einen seiner Ansprechpartner, den Cardinal Senensis (gemeint ist Francesco Piccolomini, der Neffe des - einige Jahre zuvor verstorbenen - Papstes Pius II.) antreffen könnte. Straub schreibt: Der cardinal Senensis ist nit zu Rom des sterben $\beta$ halp und underwegen, alß ich gen Senis kam, erfur ich, daz er was von der straß 6 miln zu Pyentz; bin zu $i[\mathrm{~h}] m$ vom weg geritten, hab $i[\mathrm{~h}] m$ geantwort des pfaltzgrafen brief, hatt mich wol entpfangen, erlich gehalten, essen und trincken geben. Pyentz - die Stadt der Piccolomini - heißt heute Pienza.

Der - als Deutschlandfreund weithin bekannte - Kardinal hatte ein offenes Ohr für Straub. In Bezug auf die gewünschte Confirmatz, also die Bestätigung des Vertrags zwischen Kloster und Stadt, klang er optimistisch. Straub hatte aber noch einen weiteren Wunsch im Gepäck, der nicht zu seinem offiziell formulierten Auftrag gehörte: Offenbar hatte er dem Heilbronner Rat versprochen, sich für einen Ablassbrief einzusetzen - zur Finanzierung eines neuen Chores für die Heilbronner Stadtkirche St. Kilian. Piccolomini konnte Straub in dieser Hinsicht keine allzu großen Hoffnungen machen, ihm selbst seien die Hände gebunden, dan der papst hab $i[\mathrm{~h}] m$ furgesetzt, kein volkomen applas zu geben - jedenfalls nicht in dieser Größenordnung. Doch ermutigte der Kardinal den tiefbeeindruckten Syndikus, es mit einer supplicatz, also einem Bittgesuch, direkt beim Papst zu versuchen. Bevor sich Straub wieder auf die Reise machte, stattete ihn der Kardinal mit mehreren Empfehlungsschreiben aus, unter anderem einer furdernuß an ein bischoff zu Rom und an ein procurator $z u$ Rom $^{116}$.

Die weitere Reise erwies sich als noch beschwerlicher als erwartet. Straub klagt: Das pferd, das mir Waldenberg kauft hatt, ist ein gantz ein schelm ${ }^{117}$, habß hinder mir mußen laßen dry tag reyß von Rom und hab mit marterern gen Rom komen. Die letzten rund 120 Kilometer musste der mittlerweile immerhin rund siebzig-

114 Schreiben vom 1. November 1485; ebd., U 1366 o, S. 301 f.

115 Vgl. Carl Ruland, Art. „Bibra, Kilian von“, in: Allgemeine Deutsche Biographie, hg. durch die Historische Commission bei der Königlichen Akademie der Wissenschaften, Bd.2, Leipzig 1875, S. 613.

116 Schreiben vom 1. November 1485; UB Heilbronn (wie Anm. 20) U 1366 o, S. 302.

117 Hier ein von einer tödlichen Krankheit befallenes Tier; vgl. den Artikel ${ }^{1}$ Schelm, in: Deutsches Rechtswörterbuch, Bd.XII, Sp.421-423. 
jährige Straub somit zu Fuß reisen. Am 25. Oktober 1485, gerade einmal anderthalb Monate nachdem ihm der Auftrag erteilt worden war, erreichte der Notar die Ewige Stadt.

Schnell wurde Straub klar, dass dies nicht die letzte Hürde seiner Mission sein würde: Eß stet ubel zu Rom, schrieb er nach Hause, gott kriegt mit $i[\mathrm{~h}] n$ und die welt, $i[\mathrm{~h}] \mathrm{r}$ nachpur und auch alle anderen ${ }^{118}$. Nicht minder schlimm war, dass keiner der Kontaktleute, die man Straub zu Hause anempfohlen hatte, verfügbar war: Wer nicht an der Pest verstorben war, weilte nicht in der Stadt: der general[prior der Karmeliter] ist noch nit zu Rom, noch der munch keiner, an die ich brief han. Selbst derjenige, bei dem Straub hätte wohnen sollen, war tot, weshalb der Syndikus im Gasthaus zu der glocken abgestiegen war. Laßend euch min hußfrau und min gut befolhen sein, bat der die Heilbronner Ratsherren - es ist das einzige Mal, dass wir von Straubs Ehegattin lesen ${ }^{119}$; die ungewohnte private Bemerkung lässt erahnen, dass Straub ernsthaft in Sorge auch um die eigene Zukunft war. Aber er ließ sich nicht unterkriegen: der anfanck ist gut geweßen, end soll beßer werden, notierte er, bevor er seinen Brief abbrechen musste, weil der Bote nicht länger warten wollte $e^{120}$.

Straub entschloss sich nun, diejenigen Männer aufzusuchen, die ihm der Kardinal von Siena benannt hatte: Dr. Veit Meller, einen aus Deutschland stammenden Prokurator ${ }^{121}$, dann - gemeinsam mit diesem - den abreviatori, der suplicatz macht (Cock?). Obgleich es sich also um einen für derartige Eingaben zuständigen päpstlichen Kanzleibeamten handelte, war Straub mit der Arbeit des ihm zugewiesenen Abbreviators nicht zufrieden: Die aufgesetzte Bittschrift an den Papst missfiel

118 UB Heilbronn (wie Anm. 20) U 1366 o, S. 302.

119 Parallel dazu wird Maria in der „Straubbibel“ als „Hausfrau“ des Josef bezeichnet.

120 UB Heilbronn (wie Anm. 20) 1366 o.

121 Der umtriebige Meller begegnet in den unterschiedlichsten Positionen: Laut Sinnacher war „Veit Meller, Doctor der Dekreten, von Memmingen“ zum Dekan von Innichen gewählt worden; am 3. August 1483 wird er vom Chorkapitel ermahnt, dort auch zu erscheinen, was aber nicht geschah. 1495 wird er Domherr zu Augsburg und bald darauf zu Freising, schließlich ebendort Propst zu St. Veit. Er starb zu Augsburg am 24. November 1517, in einem Alter von 73 Jahren, vgl. Franz Anton Sinnacher, Beyträge zur Geschichte der bischöflichen Kirche Säben und Brixen in Tyrol, Bd.3, Brixen 1823, S. 491. Später bedienten sich auch die Schwäbisch Haller der Unterstützung Mellers in Angelegenheiten der Klosterreform, vgl. Christian Kоцв, Zur Geschichte der Franziskaner in Hall, in: Württembergisch-Franken NF 4 (1892) S. 1-24, insbesondere S. 16. Als Anwalt in Augsburg war Veit Meller für Schwäbisch Hall auch im Streit mit Comburg um die Selbständigkeit der Pfarrei von St. Michael tätig, vgl. Johann Herolt, Chronica zeit- unnd jarbuch vonn der statt Hall, bearb. von Christian Kolb (Geschichtsquellen der Stadt Hall, Bd.1; Württembergische Geschichtsquellen, Bd.1), Stuttgart 1894, S.108f.; Kolb, Zur Geschichte der Franziskaner (wie soeben), S. 20 f. 1501 wird Meller Rat Georgs des Reichen von Bayern; 1510 ist er „litterarum apostolicarum sollicitator, familiaris“ Papst Julius’ II., vgl. hierzu Heinz Lieberich, Die gelehrten Räte - Staat und Juristen in Bayern in der Frühzeit der Rezeption, in: Zeitschrift für Bayerische Landesgeschichte 27 (1964) S. 120-189, 142, 148, 177. 
Straub derart, dass er noch eine zweite anfertigen ließ. Mit dieser im Gepäck wandte er sich an den refferendaren, das ist der bischof, dem der cardinal geschrieben hat; der vertröstete Straub zunächst, er solle uf aller seln tag umb vier aurn zu ihm kommen, dann werde er sich alles anhören und furbas min brief und meinung dem bapst getreulich furbringen ${ }^{122}$. Doch damit war noch nicht viel gewonnen.

Die Referendare hatten zwar direkten Zugang zum Papst und mussten diesem die von Ihnen akzeptierten Eingaben vorlegen. Die Zahl dieser entgegengenommenen Suppliken belief sich damals allerdings auf rund $12.000 \mathrm{im} \mathrm{Jahr}{ }^{123}$. Jede Woche standen für den Papst und seine Vertreter somit mehr als zweihundert Suppliken zur Entscheidung an - natürlich fiel das Votum nicht immer wie erhofft aus ${ }^{124}$.

Am 5. November schrieb Doktor Meller an den Heilbronner Rat und zeigte sich alles andere als optimistisch, dass der Papst den zwischen Kloster und Stadt ausgehandelten Vertrag akzeptieren und bestätigen würde, entsprächen doch einzelne Bestimmungen dieses Vertrages durchaus nicht der Rechtsaufassung des Papstes. Unser $b$ [eiliger] $v$ [ater] baupst ist noch hinher vast schwer gwese, sollich und ander concordy und privilegi ze besteten, als ier von maister Niclausen des und was uns begegnet ist personlich babs underricht werdent ${ }^{125}$.

Zwar war also noch nichts erreicht, aber man staunt, mit welcher Geschwindigkeit sich Straub bis zum Papst vorgearbeitet hatte - er war ja nur gerade einmal zehn Tage in Rom. Doch so schnell sollte es nicht weitergehen. Am 18. Mai 1486 - also ein halbes Jahr später -, schrieb der Provinzial der Karmeliter von Dinkelsbühl aus an die Heilbronner: Mir ist geschriben von unsers ordens procurator zu Rom, wie er und maister Niclas merckliche muwe und arbat von unsers handels wegen gehabet hab bey unserm heiligen vatter dem bapst und doch aus ehafter ursach nichts [...] erlanget hab. Am Ende des Briefs erwähnte der Provinzial den eigentlichen Grund seines Schreibens: Nachdem ihm der Heilbronner Rat zugesagt hatte, die 34 gulden, so der obgenant maister Niclas von Rom herwider köm, zu bezalen, die ich in zerung der sachen halben dargeliben hab, pitte ich die mir [...] mit den taxen [also samt Zinsen] zu senden; daz beger ich ${ }^{126}$.

Im August 1486 - knapp ein Jahr nach Straubs Abreise - entzogen Prior und Konvent der Heilbronner Karmeliter Magister Straub die von ihnen erteilte Vollmacht an den päpstlichen Hof ${ }^{127}$. Straub, der immer noch in Rom weilte, dürfte hiervon allerdings nichts erfahren haben. Über die Dichte der Kommunikation zwischen den Heilbronnern und Straub lässt sich freilich nur spekulieren. Der nächste Brief, der erhalten ist, wurde am 9. Dezember 1486 in Rom geschrieben: Sein Verfasser, der Prokurator des Karmeliterordens zu Rom Bruder Marx Cati-

122 UB Heilbronn (wie Anm. 20) U 1366 o (gegen Ende).

123 In den meisten Fällen ging es allerdings um Pfründen oder Stellenanfragen.

124 Schwarz (wie Anm. 92) S.3.

125 UB Heilbronn (wie Anm. 20) U 1366p.

126 Ebd., U 1366 q.

127 Ebd., U 1366 r. 
nensis, sparte darin nicht mit Lob über meister Niclaußen, ewerm mitburger, furwar eym wachberlichen und emßigen manne, der nichts unversucht underwegen hatt gelaßen, dennoch sei seine Mission gescheitert: Uns ist geantwurt worden durch unsern heiligen vatter den bapst, das nit not sy eine ander bestetigung des romischen bepstlichen stuls des, so wir von unserm orden vormals erlangt und erworben hond; dasselb so ir erlangt hond; dan furwar, so es euch einmal verluben und zugeben ist, durch den selben orden nit mag wyderruft werden ${ }^{128}$. Tatsächlich ging es dem Papst darum, keinen Vertrag zu bestätigen, durch welchen eine Kontrolle geistlicher Institutionen durch weltliche festgeschrieben würde. Catinensis hob hervor, dass der Misserfolg keinesfalls einer versumnuß meister Niclaußen zugeschrieben werden dürfe, der siner arbeyt oder gelt nit gespart hatt, sondern allein einem zuzuschreiben sei: dem bapst, der [...] nit horen wolt ${ }^{129}$.

Man kann fast vermuten, dass Straub dieses Schreiben veranlasst hat, um seine Geldgeber zu beruhigen. Derweil gab er sich aber noch nicht geschlagen. So erhielt er schließlich eine am 7. April 1487 aufgesetzte Urkunde Papsts Innozenz VIII., in welcher dieser seinen Dilectis filiis, rectoribus et scabinis oppidi de Alprun alias Fontissalutis Heil und apostolischen Segen spendete, auch sonst recht versöhnlich klang, aber dennoch für den Fall eines Vertrags contra libertatem ecclesiasticam mit canonicis sanctionibus drohte ${ }^{130}$. In der Sache war also nichts erreicht.

Straub dürfte nun abgereist sein; immerhin war er bis zu seiner Rückkehr in Heilbronn fast zwei Jahre wegen dieser Angelegenheit auf Reisen. War dies alles vergebens? Nicht ganz! Der Papst hatte ihm nämlich ein zweites Schriftstück auf den Weg gegeben, für das allein sich der Aufwand sicherlich gelohnt hat: Eine Ablassurkunde für den Chorbau von Sankt Kilian. Die Gesamtkosten von unglaublichen 6.000 Gulden sollten auf diesem Weg finanziert werden können ${ }^{131}$.

So kam es genau umgekehrt, als Kardinal Piccolomini bei Straubs Hinreise in Pienza prognostiziert hatte: Der Vertrag ist beim Papst durchgefallen, der angeblich unerreichbare Ablass aber wurde gewährt. Die Früchte von Straubs Mühen sind zu Stein geworden; trotz Zerstörungen im Zweiten Weltkrieg ist die Kilianskirche mit ihrem Chor Wahrzeichen Heilbronns. Auch Straub konnte den Erfolg miterleben, war mit dem Chorbau doch schon bei seiner Abreise begonnen worden; als er zurückkam, standen die Mauern des von Aberlin Jörg entworfenen spätgotischen Gebäudes bereits ${ }^{132}$.

128 Ebd., U $1366 \mathrm{~s}$.

129 Ebd., U $1366 \mathrm{~s}$.

130 Ebd., U 1366 v.

131 Ebd., U 1354; vgl. Moriz von RaUch, Baugeschichte der Heilbronner Kilianskirche, in: WVjH NF 24 (1915) S. 218-254, 229.

132 Ebd., insbesondere S. $228 \mathrm{ff}$. 


\section{Letzte Jahre in Heilbronn}

Im Streit um die Vogtei des Karmeliterklosters ging Heilbronn nun andere Wege, man entschied sich, die Heidelberger juristische Fakultät um Schlichtung anzufragen. Es dauerte aber noch ein weiteres Jahr, bis ein Kompromiss gefunden war $^{133}$. Straub war derweil in anderen Rechtsangelegenheiten aktiv ${ }^{134}$. So gutachtete er 1488 in einer Streitsache, die Kaiser Friedrich III. zur Klärung an den Heilbronner Magistrat delegiert hatte ${ }^{135}$.

Und noch im selben Jahr ist er wieder auf „Dienstreise“ - diesmal zum Kaiser. Zur Sicherung des Landfriedens in Schwaben hatte Friedrich III. durch kaiserliches Mandat vom 26. Juni 1487 die Errichtung eines „Schwäbischen Bundes“136 durch Zusammenschluss der schwäbischen Reichsstände angeordnet ${ }^{137}$. Nicht alle Reichsstände waren davon begeistert, insbesondere die Ritter fürchteten um ihre Selbständigkeit; die Reichsstädte zögerten, da sie Angst hatten, in die Zwistigkeiten anderer hineingezogen zu werden, was nicht nur den Handelsbeziehungen zum Schaden gereichen, sondern auch erhebliche Kosten erzeugen konnte. Im Oktober 1487 ließ der Kaiser daher ein weiteres Mandat folgen, indem er Prälaten, Adel und Städten Schwabens bei Verlust ihrer Freiheiten und Privilegien und bei einer Strafe von 100 Mark lötigen Goldes gebot, sich unverzüglich zu vereinen ${ }^{138}$.

Als daraufhin im Februar 1488 auf dem Reichstag in Esslingen am Neckar der Schwäbische Bund endlich offiziell gegründet wurde, war Heilbronn noch immer nicht dabei. Wie kostspielig das Engagement der Stadt zu werden drohte, ließ sich an den Verpflichtungen der anderen Reichsstädte ablesen. So musste sich Schwäbisch Hall (das damals freilich größer war als Heilbronn) mit 18 berittenen Soldaten und 170 Mann zu Fuß beteiligen ${ }^{139}$. Doch der politische Druck auf die Reichsstadt am Neckar wuchs; so hatte die Versammlung der schwäbischen Stände längst beschlossen, dass neben mehreren weiteren Territorien auch angrenzende Städte, unter anderem Wimpfen und Heilbronn, aufzunehmen seien ${ }^{140}$.

Die Heilbronner sahen nur einen einzigen Weg, sich der Zwangsmitgliedschaft zu entziehen: eine Petition an den Kaiser. Sicherlich hatten die Heilbronner die

133 UB Heilbronn (wie Anm. 20) U 1366 (bb).

134 Gemäß einem - wohl im Zweiten Weltkrieg zerstörten - Gerichtsprotokoll von 1490 half Straub als Syndikus bei der Urteilsfindung und setzte die Schriftfassung auf, vgl. JäGER, Geschichte der Stadt Heilbronn (wie Anm. 84) S. 251.

135 UB Heilbronn (wie Anm. 20) U 1433.

136 Vgl. auch Horst CARL, Der Schwäbische Bund 1488-1534 - Landfrieden und Genossenschaft im Übergang vom Spätmittelalter zur Reformation, Leinfelden-Echterdingen 2000.

137 Karl Klüpfel (Hg.), Urkunden zur Geschichte des Schwäbischen Bundes (14881533), Bd.1, Stuttgart 1846, S. XIII.

138 Ebd., S. 11.

139 Ebd., S. 32.

140 Bereits am 9. Oktober 1487 in Ulm, vgl. ebd., S. 12. 
geringen Erfolgsaussichten dieses Schrittes erkannt, aber nichts unversucht lassen wollen. So wurde Nikolaus Straub - als der erfahrenste Diplomat der Reichsstadt - nach Antwerpen geschickt, wo sich Friedrich III. damals aufhielt. Doch der Kaiser interessierte sich nicht für die Belange der Reichsstadt; für ihn war der „Schwäbische Bund“ Teil seines Landfriedenskonzepts und damit wichtiges Element der mühsam vorangebrachten Reichsreform. In einem an die Heilbronner gerichteten Brief vom 18. September 1488 erklärte er daher knapp: Nikolaus Straub, euer sindicus, zeiger diß brieffs, habe versucht ihn mit allerlei Argumenten um Erlass oder zumindest Aufschub der Mitgliedschaft im „Bund von Schwaben“ zu bewegen, doch stünden dem wichtige Gründe entgegen ${ }^{141}$.

Fast zeitgleich erging ein kaiserliches Mandat an die Städte Augsburg, Konstanz, Rottweil, Heilbronn, Wimpfen und Buchhorn, in welchem den Städten - unter Androhung der Reichsacht - eine Frist von neun Tagen für den Eintritt in den Bund gesetzt wurde ${ }^{142}$. Heilbronn hatte keine Wahl. Als Militärkontingent musste die Neckarstadt nun zehn Berittene und hundert Fußsoldaten stellen ${ }^{143}$.

Am 25. Januar 1489 - rund vier Monate, nachdem Straub vor dem Kaiser gestanden hatte, war er in Heilbronn zurück. Trotz des Misserfolgs beschloss der Rat, Meister Nikolaus Straub neun Gulden zu verehren, für sein Engagement, als er von Heilbronns und Wimpfens wegen by der k.m. gewesen ist ${ }^{144}$. Offensichtlich rechnete man die Niederlage beim Kaiser nicht seinem fehlenden Geschick zu.

Straub, der es zwischenzeitlich zu einem gewissen Wohlstand gebracht hatte ${ }^{145}$, scheint sich nebenbei eine private Bibliothek aufgebaut zu haben - im späten 15. Jahrhundert ein äußerst kostspieliges und für Bürgerliche eher ungewöhnliches Unterfangen. In der Oxforder „Bodleian Library“ hat sich jedenfalls ein Exemplar des 1484 bei Peter Schöffer in Mainz gedruckten „Herbarius“, eines lateinischdeutschen Bildlexikons der Kräuter und Nutzpflanzen, erhalten, das den Besitzereintrag Nicolaus Straub ano etc. 89 trägt $^{146}$. Ein Handschriftenvergleich lässt vermuten, dass der Eintrag aus der Feder des mittlerweile alternden Heilbronner Generalsyndikus stammt ${ }^{147}$. Das Buch gelangte 1835 auf unbekannten Wegen in die Oxforder Sammlungen.

141 UB Heilbronn (wie Anm. 20) U 1501.

142 Klüpfel (wie Anm.137) S. 39.

143 Bundestag in Schwäbisch Hall, 14. April 1489, vgl. ebd., S. 62.

144 UB Heilbronn (wie Anm. 20) U 1519.

145 In einer Urkunde vom 11. Mai 1489 (ebd., U 1530) wird ein Garten von Meister Niklaus erwähnt; da „Meister“ auch als Handwerkerbezeichnung dienen kann, selbst als Betitelung für den Scharfrichter gebräuchlich war, braucht dies allerdings nicht mit Nikolaus Straub in Verbindung stehen.

146 Hierzu: Alan Coates u.a., A Catalogue of Books Printed in the Fifteenth Century now in the Bodleian Library Oxford, Bd.3, Oxford 2005, S. 1237, H-037 Signatur: RR.w.471 [RSL].

${ }^{147}$ Freundliche Auskunft von Dr. Alan Coates, Assistant Librarian, Rare Books, Departement of Special Collections, Bodleian Library, Oxford, vom 22. August 2012. 
Als der 1493 zum Prediger in der Kilianskirche neu berufene Hans Chrener in Ingolstadt noch im selben Jahr zum Doktor der Theologie promoviert wurde, war dies offenbar auch für die Heilbronner ein großes Ereignis. In den Steuerrechnungen findet sich der Eintrag: 4 gulden meister Niclaus Strube verzert gen Ingelstatt, als er dem prediger ein erung von der statt wegen $\mathrm{zu}$ dem doctoratt gethon hat ${ }^{148}$. Es könnte dies die letzte größere Dienstreise des mittlerweile wohl ungefähr achtzigjährigen Straub gewesen sein.

Straub war aber weiterhin beruflich aktiv, begegnet - wie zuvor ${ }^{149}$ - immer wieder als Notar ${ }^{150}$ und Anwalt. Aus den Jahren 1496 und 1497 stammen mehrere von Straub ausgestellte Notariatsinstrumente in einer Erbschaftsstreitigkeit zwischen dem Heilbronner Bürger Michel Hüngerlin und der Familie Hornberger/Büschler (Rothenburg, Schwäbisch Hall), unter anderem die Ende Dezember 1496 von Straub aufgesetzte Appellation Hüngerlins an das neu gegründete Reichskammergericht ${ }^{151}$.

Aus den folgenden Jahren stammen letzte notarielle Urkunden Straubs ${ }^{152}$. Vom 8. November 1498 hat sich eine von Generalsyndikus Straub angefertigte Urkundenabschrift erhalten ${ }^{153} .1500$ trat Meister Nikolaus Straub dann noch zweimal als Fürsprech auf ${ }^{154}$, soweit nachweisbar zuletzt am 11. Mai in einer Zivilrechtsstreitigkeit vor dem Heilbronner Schultheißengericht ${ }^{155}$. Danach finden sich keine Lebensspuren mehr ${ }^{156}$. Straub muss also irgendwann nach dem 11. Mai 1500, somit im Alter von vermutlich über 85 Jahren verstorben sein.

\section{Einblicke in die „Straubbibel“}

Abschließend sollen einzelne Passagen aus der Evangelienübersetzung des Nikolaus Straub einen Eindruck von der Übersetzungsleistung des Notars liefern ${ }^{157}$.

148 Eintrag zum 6. Oktober 1493 in: UB Heilbronn (wie Anm. 20) U 1709.

149 19. August 1490, ebd., U 1582; 20. August 1490, ebd., U 631 b; in den Beiakten zu einem Prozess vor dem Reichskammergericht findet sich ein Appellationsinstrument des Notars Nikolaus Straub aus Leonberg aus dem Jahr 1493 (damals noch an den Kaiser; ausführlich U 362), vgl. Brunotte/Weber (wie Anm. 29) S. 139.

150 So 1495, vgl. UB Heilbronn (wie Anm. 20) U $1535 \mathrm{k}$.

151 Ebd., U 1775 a; Reichskammergerichtsakten HStAS C 3 H Nr. 2159 (U 470 ff.), BRUnotTe/Weber (wie Anm.29) S. $426 \mathrm{f}$.

152 Notariatsinstrument vom 5. Dezember 1498, UB Heilbronn (wie Anm. 20) U 1582 f; notarielle Beglaubigung vom 5. April 1499, ebd., U 1816 a.

153 Ebd., U 15351.

154 Ebd., U 1842 c (ohne genaues Datum).

155 Ebd., U 1848.

156 Vgl. etwa Band 3 des Heilbronner Urkundenbuchs.

157 Weitere kurze Passagen finden sich bei: Zimmermann (wie Anm.1) S.71*-76* (Matth. 2,1-12; Joh. 2, 1-11; Matth. 9, 1-9; Luk. 2, 1-14; Joh. 5, 1-9). Bei Jochen SPLETT 
Um die Lesbarkeit und Verständlichkeit vor allem theologisch ungeübten Lesern zu erleichtern, wurden hierfür vornehmlich allgemein bekannte Bibelstellen ausgewählt: Das Vaterunser in den Fassungen von Lukas und Matthäus sowie die Weihnachtsgeschichte aus diesen beiden Evangelien. Um den Eindruck aber nicht allzu sehr zu verzerren, sind die Textpassagen jeweils in einen weiteren Kontext gestellt. Die Übertragung erfolgte, soweit möglich, buchstabengetreu; zeitübliche Abkürzungen wurden aufgelöst, ansonsten erfolgt eine Erklärung des abgekürzten Wortes in eckigen Klammern. Unleserliche Wörter sind mit einem Fragezeichen gekennzeichnet. „U“ und „V“ sowie „i“ und „j“ wurden im Zweifel der heute üblichen Schreibung entsprechend gesetzt; wo Straub ein „w“ (= vu) für „wu“

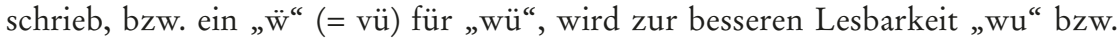
„wü“ gedruckt (z.B. „antwurt“ statt „antwrt“, „wunderten“ statt „wnderten“, „würd“ statt „ẅrd“). Die Zusammen- und Getrenntschreibung wurde beibehalten; als - auch in Straubs Urkunden vorkommende - Besonderheit fällt hierbei das häufige Zusammenschreiben der Präposition „in“ mit dem jeweils nachfolgenden Wort ins Auge („Inmitter naht“, „Inden“, „Ingalilea“ usw.). Die Zeichensetzung mit Virgeln wird der Handschrift entsprechend gezeigt, jedoch, wo zur besseren Lesbarkeit nötig, ein Komma, Punkt oder Doppelpunkt eingefügt. Zudem werden Satzanfänge durch Großschreibung gekennzeichnet.

\section{Rund um das Vaterunser}

a) Lukas 11, 1-28

\section{[Das Vaterunser]}

Und eß geschah, alß Jhesus bett/ an einer stat/ und als er uff hort zu betten/ sprach einer außer sinen Jüngern/ herr/ lere unß bettn/ als Johannes hatt gelert sein Jünger/ und er sprach/ zü den/ so Ir bettend/ so sprechend/ vatter unßer/ din nam werd heilig/gemacht/ din reich komm un $\beta$ zil/ gib unß allen tag unser deglich brot/ und laß uns abe/ unßer sünde/ [113 r] gleich weiße/ und wir ablaßn/ alln den/ die un $\beta$ schuldig sind ze tond/ und fur uns/ nit In versubnust.

Und sprach zu den/ welcher under euch/ wirt haben ein fründ/ und wirt/ zu dem gen/ Inmitter nabt/ und wirt zu dem sprechen/ frund lib mir drüw brot/ dann mein frunt/ ist komen vom weg zü mir/ und Ich bon nit/ daz Ich für In lege/ der antwert furbas/ und sprech/ biß mir nit leidßam/Itcz ist min tüer beschlossen/ und min kint sind bey mir/ In min schlafkammer/Ich mag nit aufsten/ und dir geben/ und der/

(Hg.), Das hymelreich ist gleich einem verporgen schatz in einem acker ... - die hochdeutschen Übersetzungen von Matthäus 13, 44-52 in mittelalterlichen Handschriften, Göppingen 1987, S.197f., sind Bl.24v. und 25 r. der Straub-Übersetzung abgebildet (mit dem Text zu Matth. 13, 35-55). Herrn Dr. Christoph Mackert, Leiter des Handschriftenzentrums der Universitätsbibliothek Leipzig, danke ich herzlich für das Verfügbarmachen der Handschrift. 
ob er wirt kloppfend bleyben/ sag Ich euch/ ob er dem nit wirt geben/ und aufsten/ dar umb daz er sein frunt ist/ so stet er doch auf/ von wegen sein $\beta$ emßigen gebetez wegen/ und unruwe/ und wirt Im geben/ waß er noturftig ist/ und Ich sag euch/ begern so wirt euch geben/ süchend so werdnd Ir finden/ kloppfend an/ so wirt euch aufgeton/ dan ein Iglicher der begert/ der nimpt/ und wer sücht der wirt finnden/ und dem kloppfenden wirt auf geton/ wer aber ist außer euch/ der vom vatter begert brot/ wirt er Im nit geben ein stein/ oder der begert ein fisch/ wirt er Im nit für den fisch geben ein schlangen/ oder ob er wirt begern ein aye/ wirt er Im nit bieten dafur ein scorpion/ ob er zu den dreyen sachen und begerungen sprach nein/ hierumb ist eß/ so Ir boße sind/ daz ir erkennend die guite dinge/ zü gebend ewern sün/ wie fil mer/ ewer vatter vom himmell/ wirt geben/ ein giiten geist/ [113v] den die In bitten.

\section{[Jesus und die bösen Geister]}

Und Jhesus warff auß ein teufell/ der waß ein stumm/ und die schar wundert sich/ und als Jhesus den herauß bet geworfen/ rett der stumm/ ettlich außer In/ sprachen/ er wirfft die teufel auß/ In beelzabup dem fursten/ der teuffell/ die andern versuichten In/ begerten ein zeichen von Ime/ er als er sab/ Ir gedechtnüß/ sprach zu In/ ein Iglich reich/ daz Insich selb $\beta$ geteilt ist/ wirt zergen/ und daz bauß wirt fallen [?] auf das/ hauß/ ist das dann/ daz der teufel Insich selb $\beta$ geteilt ist/ wie wurt dann sein reich steen/ dann Ir sprechend mich außwerfen die teufel/ Inbeelzebub/ und ist daz/ daz Ich auß wirff die teufel Inbeelzepupp/ wie sein/ Inwem werfen sie dann auß/ darumb werden sie euch urteilen/ aber/ so ich außwirff die teufel/ In der crafft dez heiligen geistes/ fur war/ wirt Ineuch komme daz reich gottes/ so der starck gewappett bebüt sein hof/ so sind alle ding In frid/ die er besitczt/ Ist eß aber/ daz ein stercker Im kompt/ In übirwint/ so nimpt er alle seine wafen/ hin/Inden er getrawt hatt/ und außteilt seinen raub/ wer nit mir ist/ der ist wider mich/ und wer nit mit mir sammelt/ der wirt auß ströwen/ so der unrein geist wirt auß geen vom menschen/ so wandelt er durch die dürren stett/ und sücht in aber [?] und so er nit fint/ spricht er/ Ich werd wider kummen/In mein hauß/ da von Ich bin außgangen [114 r] und so er ist komen/ so fint er daß bauß gereinigt/ mit besemen/ und so get er/ und nimpt/ zu Im/ ander syben geist/ böser dann er/ und so sie In sind gangen/ wonen sie dal und sind die lettschsten deß menschen/ bößer den ersten. Und es geschab/ alß er die red tett/ bub ein fraw/ von der schar/ Ir stimm auf/ und sprach zu Jhesus/ selig ist der leip/ der dich getragen hatt/ und die brust/ die di̊ gesogen hast/ Jhesus sprach/ furwar/ selig sind die/ die hörnd daz wort gottes/ und behüten das.

Markant ist hier die Abweichung des Vaterunsers von der heute gewohnten Form. Sie entspringt einer divergierenden Überlieferung bereits in Straubs Vorlage. Ursprünglich bringt Lukas das Vaterunser knapper als im Matthäusevangelium (Matth. 6, 9-13). Diese knappere Version dürfte der anfänglichen, auf Christus 
zurückgehenden Form entsprochen haben. In zahlreichen Bibelhandschriften erfolgte dann eine (zumindest teilweise, unterschiedlich weit gehende) Anpassung des Lukas-Vaterunsers an die - vermutlich im Rahmen der Liturgie der Kirche ausgebaute Version im Matthäusevangelium ${ }^{158}$. Zum Vergleich sei daher auch die zweite Stelle des Vaterunsers in der Version von Straub gebracht:

b) Matthäus 6, 1-15

\section{[Vom Almosengeben]}

Habend acht/ merckend/ und bütend euch, daz Ir Icht ewr gerechtikeit tond/ vor den menschen/ umb deß willen, das Ir von In werdend an gesenhen/Ir habend anders ewern lone/ nit bey ewerm vatter/ der Inn himmeln ist/ hierumb, wann $d u$ geist das allmißßen, so solt $d u$ daz vor der nit auß schreyen/mit eim herhorn/ als die gleyßner tuind/In Iren sammelungen/ und an den gasßen/ umb daz/ sie geert werden/ von den menschen/fur war sag Ich euch/ sie haben Iren lon entpfangen/ aber so $d u$ das allmuißen gyst/ so gib $\beta$ also/ daz din linck hant/ nit wis $\beta$ waz tüwe din gerecht hant/ umb deß willen/ daz dein allmüßen sey verborgen/ und din vatter, der daz verborgene sicht/ wydergellt dir $\beta$.

\section{[Vom Beten. Das Vaterunser]}

Und so Ir bettend/ sullend Ir nit sin/ als die traurigen gleißner/ [10 r] die liephaben/ stend/In Iren sammlungen/ und Inden biegeln der gasßen/ und betten/ daz sie gesenhen werden von den menschen/ furwar sag Ich euch sie haben genommen Iren lone/ aber so du bettst/ gang Inden schlauf kammer/ und beschleuß dein türe/ bitt dein vatter beimlich/ und din vatter der daz beimlich sicht/ wider lont dirß/ aber so Ir bettend, so sult Ir nit fill reden/ so die heiden tond/ furwar sie meind sie werden ber bört In Ir fil red/ Ir sullend euch In nit gleich machen/ furwar ewr himmelischer vatter/ weyß waz euch noturfftig sey/ e/ dann Ir bittend// hierumb so werdend Ir also betten:

Vatter unßer/ der du bist Inn bimmeln/ gebeiligt werd din nam/ zü komm unß dein reich/ din will werd/ als Im himmel/ und In der erden/ unßer teglich brot gib uns heut/ und vergib uns unßer schuld/ als und wir ablaßen/ und vergeben/ unßern schuldnern/ und fur uns nit In/ Inversüchnust/ besonders erlöß unß von übel/ furwar ob Ir werdend ablaßen/ den menschen Ir sünde/ so wurt ewr himmelischer vatter euch auch ablaßen/ ewr sünde und schulden.

Die Ähnlichkeit mit der dem heutigen Leser vertrauten Lutherübersetzung ist hier - wie an mehreren Stellen in Straubs Text - so groß, dass sich dies allein mit der beiderseitigen Verwendung derselben lateinischen Vorlage kaum erklären lässt.

158 Vgl. etwa Ernst Lautenbach (Hg.), Lexikon Bibel Zitate, Auslese für das 21. Jahrhundert, München 2006, S. 37. 
Math

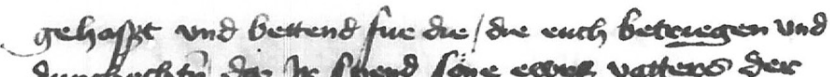

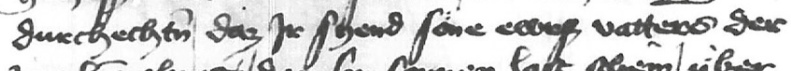

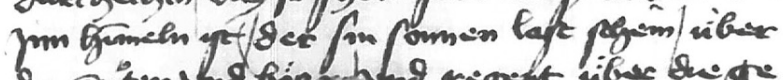

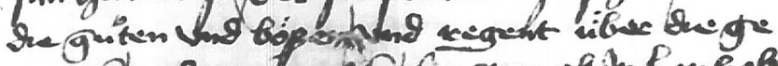

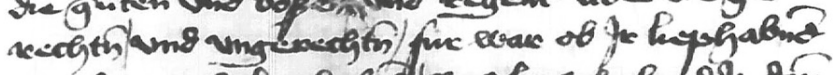

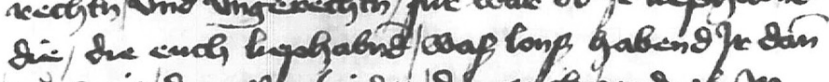

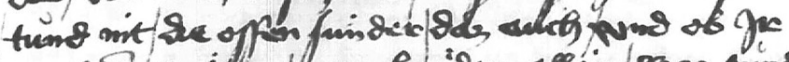

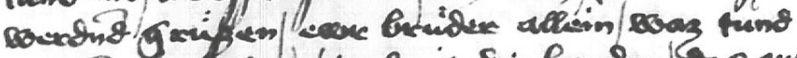

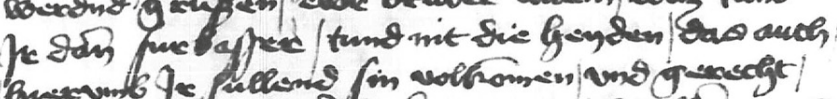

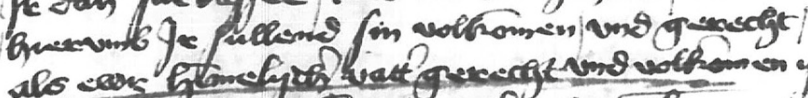

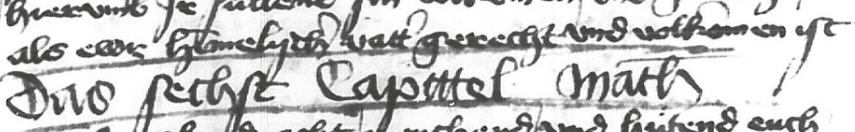

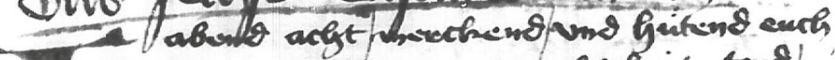

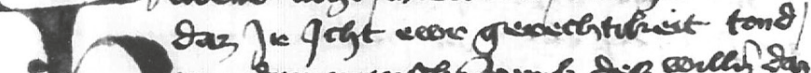

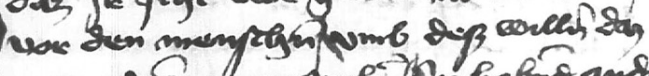

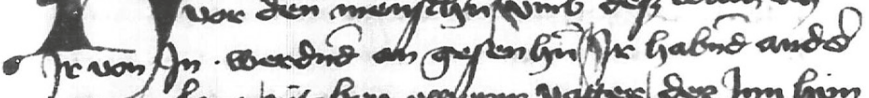

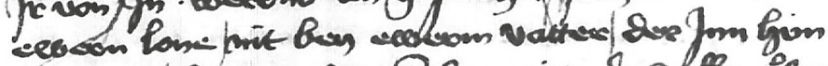

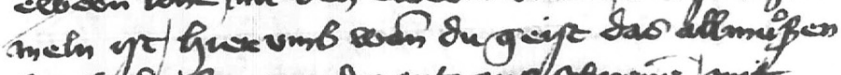

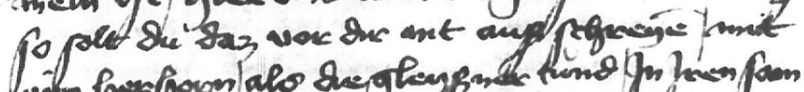

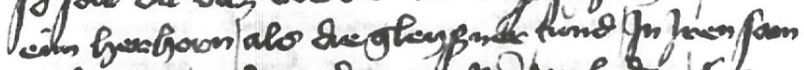

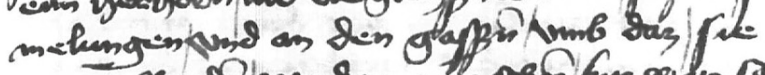

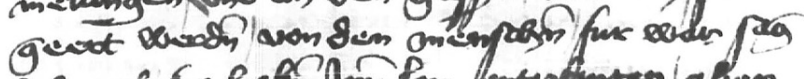

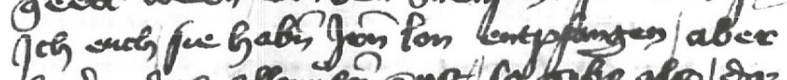

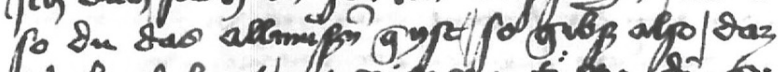

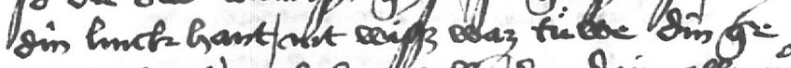

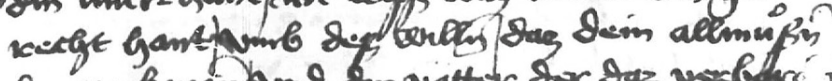

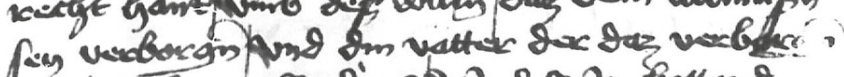

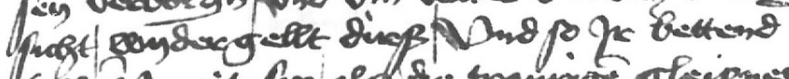

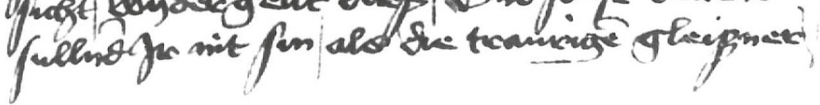

Abb.5: Beginn von Kapitel 6 des Matthäus-Evangeliums im Manuskript der Übersetzung von Nikolaus Straub (Universitätsbibliothek Leipzig, Ms 35, Bl.9v). 
Sollte Luther also die „Straubbibel“ gekannt haben? In Anbetracht der Tatsache, dass es von Straubs Übersetzung - nach allem was bekannt ist - nur ein Exemplar gegeben hat, erscheint dies so gut wie ausgeschlossen. Sollten Luther und Straub also ein gemeinsames Vorbild gehabt haben? Straub betont, wie oben erwähnt, seine Übersetzung ohne Kommentare und Hilfsmittel angefertigt zu haben, woran auch Experten wie Handschriftenkenner Erich Zimmermann keine ernsthaften Zweifel hegen ${ }^{159}$. Allgemein gilt Straubs Übersetzung als sehr selbständig ${ }^{160}$. Es ist allerdings anzunehmen, dass es bereits im 15. Jahrhundert eine mündliche deutschsprachige Überlieferungstradition für wichtige Bibelpassagen gab: Bedeutende Bibelstellen wurden den Christen zum besseren Verständnis auf Deutsch vermittelt und so naturgemäß auch weitergetragen. Dass es vor allem zentrale Bibelpassagen sind, in denen sich Straubs und Luthers Formulierungen einander annähern, lässt sich eindrücklich am Beispiel der Weihnachtsgeschichten (nach Lukas und Matthäus) in Straubs Übersetzung illustrieren. Liest man diese in einem weiteren Textzusammenhang, dann erinnert dieser gar nicht so sehr an die vertrauten Formulierungen, sehr wohl aber an die Kernsätze der Weihnachtsgeschichte:

\section{Die Weihnachtsgeschichten im weiteren Kontext}

a) Lukas 2

Und eß geschah Inden tagen/ geng auß ein gebott vom [92 v] keyßer augusto/ das die gantcz wellt/ wird angeschriben [?]/ Die an schreibung ist zu ersten beschenhen/ vom richter cyrino/ der stat syrie/ und sie gengen alle/ das sie glöpten und verjehen/Iglich Insin statt/Joseph stig auf von galilea/ von der stat nazareth/Indie Juttschen statt davitcz, die dan heyst betthlehem/ dar umb/ dan Joseph gehört zum hauß und haußgesind davitcz/daz er verjeb/ und glöpt/ mit maria siner/ schwangern vermähellten haußfrawen/ und eß geschach/ als sie da waren/ sind erfullt die tag/ daz sie gebere/ und sie gebar/ Iren erstgebornen son/ und wickelt den Indie tüchlin/ und neigt In nider Indie krüppen/ dann sie hett sunst dhein statt/ under dem vorschoppff/ und die hirten waren Inder selben gegen/ wachten/ und butn die wacht/ der nacht/ über Ire schäffereye/ und nimm war/ der engell deß herren/ stond bey den/ und die clarbeit gottes/ ummschin die/ und sie forchten sich/ mit großer forcht/ und der engell sprach zu In: forchtend euch nit/ nimm war/ ich verkund euch/ ein große fröd/ die da wirt allem folck/ dann unß ist heut geborn der beilmecher/der da ist xptus [= Christus]/der herre/ Inder stat david/ und zu einem zeichen/Ir werdend finden ein Jungß Ingewickellt/ In tüchlin/ und gelegt Indie krüppen/ und bald mit dem engell/ beschab und quam zü ein ander/ ein große mengin der himmelischen ritterschafft/ die got lopten [93 r] und sprachen/ ere sey

159 Zimmermann (wie Anm. 1) insbesondere S. 72\% ff.

160 Vgl. die oben (ab Anm. 2) genannten Lexika-Einträge. 
got Inden obristen tronen/ und auf dem ertereich/ sey der frid den menschen/ ein $\beta$ guiten willens/ und eß geschach/ alß die engell hinweg schieden/ Inn himmell von den birten, retten die birten zu einander/ so/ gend/ wir hin gen bethlebem/ und senhen das wort/ daz worden ist/ daz der herr gemacht und uns erzögt hatt/ und quamen eylend/ und fannden mariam/ Joseph/ und das kindelin/ ligend Inder krüppen/ und so sie das saben/ erkanten sie von dem wort/ das In gesagt was/ von dem kind/ und all die das gehort/ hetten/ wunderten sich/ über die ding/ die dann die birten zu In gesagt hetten/ aber maria die behielt die ding alle/ und beschloß die In Ir hertcz/ die hirten quamen widerumb/ erten/ und lopten/ gott/ Inallen dingen/ die sie gehort und gesenhen hetten/ alß eß gesprochen ist zů Inen.

Und als volbracht sind acht tag/ daz beschnitten wirde das kint/ ist sin nam genempt/ Jhesus/ der selb nam also benempt ist/ vom engell/ e dann das kint enpfangen ward Im leip/ und nach dem/ und die tag der reinigung marie/ erfult sind/ trugen sie Jhesum nach der gesätcz/ moisi/ In Jherusalem/ das sie den dem heren entwürten/ oppferten/ und geben/ als eß geschriben ist/ Inder gesatczt/ deß herren/ ein Iglich gebürt nach dem geschlecht/ ein kneblin/ oder mennlin/daz dann auf tuit die ersten gepurt siner muter/ der wirt gesagt beilig gemacht oder gesegnatt/ dem berren [93v] und das sie gaben/ das oppfer/ nach dem und gesaczt ist/ Inder gesatczt deß herren/ ein par turtel tauben oder sunst zwo Junge tauben/ und nimm war ein mensch waß In Jhrlm. [= Jherusalem]/ deß nam was symeon/ und der mensch was gerecht/ und forchtsam/ und wartet auch die tröstung/ und erlösung I ßrael/ und der heilige geyst was In Ime/ und nam ein antwurt/ vom heiligen geist/ daz er den tod nit würd versüchen/ eß wer dann/ daz er vorbin sehe xpm. [= Christum] deß heren/ und er quam Im geist Inden tempell/ und als sie Infurten das kint Jhesum/ sin vatter und muiter/ dar umb daz sie/ mit Im und für In/ tetten nach gewonheit der gesatczt/ und symeon nam Jhesum Insin arme/ und sprach/ woll got/ und sagt: Herr nun send din knecht/ nach deme wort/ Im fride/ dann min augen/ haben gesenhen/ din heile/ das du gemacht hast/ vor angesicht/ all volck/ ein liecht zui offenbarung/der fölcker/ und zu ere din $\beta$ folck $\beta$ I $\beta$ raell und sin vatter und sin miter/ wunderten sich über die ding/ die von Im gesagt warden. Und symeon sprach den woll und sprach zu maria siner muiter: Nimm war Jhesus/ ist gesetczt/ zu eim fale/ und auferstenung fil In Ißrael/ und deß zu eim zeichen/ so wirt Jhesu/ widersprochen/ und din sele/ die auch sin ist/ wirt durch gen ein schwert/ daz die gedenck werden geoffenbart/ auß fil bertzen.

Und eß waß annal ein wyßsegin/ ein dobter phanuel/ vom geschlecht asser/ die was furgangen/Infil [94 r] tagen/ und hett gelebt mit Irem man syben Jare/ nach Ir Jungfrölikeit/ und bleib ein wittwe/ biß zi vier und achtig Jarn/ und die geng nit vom tempell/ besonder sie dient got/ tag und nacht/ mit fasten/ und betten/ dieselb quam zü der selben stond/ auch Inden tempell/ verjah dem herrn Jhesu/ und rett von Im/ allen den/die dann beeten erlösung Jbrlm. [= Jherusalem]/ und als sie alle ding volbrachten/ nach der gesatcz moysi/ sind sie widerumb kommen/ Ingalileal In Ir stat nazareth/ aber daz kint wuchs/ und wart gesterckt/ fol wißheit/ und 
die gnad gottes was In Ime/ und sin vatter und miter/ gengen alle Jar/ gen Jbrlm [= Jherusalem] auf den hobzittlichen tag austern. Und als Jbesus was worden zwölff Jar alt/ als sie auf gengen/ gen Jbrlm. [= Jherusalem]/ nach gewonbeitt deß hobzittlichen tages/ und als die tag volbracht waren/ und widerumb gengen/ blib das kint Jhesus/ In Jrblm. [= Jherusalem]/ und sin vatter und miter wisten dar umb nit/ doch meinten siel er wer ettwan Ineime gesellschafft/ und als sie ein tag-reiß gangen waren/ sichten sie In widerumb under sinen fründen/ und erkanten/ und so sie In nit funden/ gengen sie widerumb In Jbrlm. [= Jherusalem]/ und suchten Jhesum/ und eß geschab/ das sie In nach dryen tagen/ fanden Im tempell/ sitczen In mittell/ der lever/ die horten Jbesum/ und fragten In/ und all die In borten/ erschracken von siner fursichtikeit/ [94v] und antwurten/ und so sie daz saben/ wunderten sie/ und sprach sin muiter/ zu Jhesus: Son/ waß hast du unß also geton/ nimm war/Ich und din vatter/ haben dich mit traurn gesucht/ und er sprach zu In: Was ist daz/ daz Ir mich gesucht hond/ habend Ir nit gewist/ das Ich miß volbringen/ sachen die minem vatter zü gebörn/ und $\sin \beta$ willen $\beta$ sind/ und sie verstonden das wort nit/ das er zu In rett/ und er geng mit In al/ von Jbrlm. [= Jherusalem]/ und quam gen nazareth/ und was den undertenig/ und sin miter bebielt die wort alle/ verbarg die In Irem hertczen/ und Jhesus nam zil/ Indem alter/ und weißheit/ In gnad vor got und den menschen. [Es folgt das 3. Kapitel].

b) Matthäus 1,18-25 und 2

Und allso ist die gepurt xpi [= Christi] geweßen und zu gangen/ alß maria die muiter Jhesu waß vermähelt und glopt Joseph Irem sponsen/ E dann/ und sie/ zu samen [?] quamen Ist maria gefunden/ habend In Irem leip/ vom heiligen geist/ Joseph aber Ir man/ so er gerecht waß/ wolt sie nit In fürn Insein hauß/ besonders er wolt sie heimlich verlaßen/ und als er diße sach allso bedacht/ nem war der engel deß herren erschin Joseph und sprach: Joseph/ ein son davitcz/ forcht dich nit/ ze nemen maria dein gemabel/ dann daz In Ir geporn ist/ ist vom heiligen geiste/ aber sie wirt gebern ein sone/ und di wirst nennen sein namen Jhesus/ fur war er wirt beil machen/ sein folck von Iren sunden/ aber das ist alles beschenhen/ das erfullt würde/ daz gesprochen ist vom herren/ durch den propheten sprechend: nim war die Jungfraw wirt habn In Irem leip/ und wirt gebern ein son/ sin nam wir genempt emanuel/ daz ist so vil gesagt/ got bey unß/ also stond auf Joseph/ vom schlauff/ und tett als Im der engel deß herren gebot/ und nam bin sinen gemabel/ und erkant sie nit/ biß sie gebar Iren erstgebornen sone/ und nampt sein namen Jhesus.

Daß ander Capittell:

Do nun Jhesus/ allso geboren waß zu bethlehem/Jude/ Inden tagen herodis deß küngs/ nim war/ die weyßen meister oder kunig quamen von orient/ gen Jherusalem/ und sprachn: wo ist der künig der Juden/ der geporn Ist [4 r] dann wir haben gesenhen sin stern/ Inorient/ und wir kommen/In anbetten/So herodes der küng daz hort/ ist er betrubt/ und als folck zu Jherusalem mit Im besonders die herodi günstig waren/ und sammelt herodes/ all fursten der priester/ und die geschrift wei- 
ßen deß folcks/ und lernt von In fragend/ wo xpus [= Christus] solt geporn werden/ und die sagten Im Inbethlahem Judel dann allso ist geschriben durch den propheten/ und du bethlehem ertrich Judal du bist doch nit die minnst Inden fursten Judal dann auß dir wirt geen ein fürer/ der wirt regiern mein folck Ißrabel/ also berufft berodes heimlich zi Im/ die meister/ und weisen kunig/ und lernt mit fleiß von In/ daz zeit/deß stern $\beta /$ der In erschinen waß/ und sant die Inbethlehem/ und sprach/ gend hin/ und fragend mit fleiß vom kinde/ und so Ir $\beta$ habend gefunden/ wider kündend mirß/ daß Ich komm und In auch anbett/ die selben als sie den künig gehorten/ giengen sie hin/ und nim war/ der stern den sie gesenhen hatten Inorient/ gieng In vor/ so lang/ biß er quam do daß kint waß/ stond ober dem bau $\beta /$ alß sie den stern saben/ sind sie erfrowet/ mit fast groser fröd/ und giengen In das bauß/ funden das kint/ mit und bey maria seiner miter/ und fielen nieder fur sich/ betten In an/ und tetten auf Ir schetcz und gauben/ oppferten Im die gaub/golt/ weyrauch und mirr/ und so sie die antwurt enpfiengen Im schlauf/daz sie nit widerumb quemen $[4 \mathrm{v}]$ zu herodil sind sie ein andern weg widerumb kommen In Ir gegen/ und alß sie binweg giengen/ nim war/ der engel deß herren/ herschin Joseph/Im schlauf/ und sprach/ stand auf/ und nim bin daz kint und sin miter/ und floub Inegypten/ und biß da/ so lang und Ich dir wird sagen/ dann e $\beta$ ist kunfftig/ und binvor/ daß herodes wirt süchen daz kint/ zü verhern und ertöten daz selbel der selb Joseph stond auf/ nam bin das kint und sin miter/ und Inder nacht/ fur er bin Inegypten/ und bleib da/ biß zum tode herodis/dar umb daß erfüllt wird das gesagt ist vom herren durch den propheten/ sprechend/Ich hab berüft auß egypto minen sone. Dann herodes so er sabe, das er betrogen war/ von den weißagen und kungen/ ist er fast erzürnt/ und sant/ her tot/ alle kint Inbethlehem/ und In allen enden von der zeit zweyer Jar/ und dar under/ nach dem und er die zit erfarn hett von den weißagen/ dann ward erfult/ das gesagt waß/ durch den propheten sprechend/ die stimm ist erbört Inder höbin weinung der kind/ und hewlung der müter/ rachel die fraw weint Ir süne/ und sie wolt nit getröst werden/ dann sie sind nit. Aber als herodes vergraben ward/ nem war der engel deß herren erschin Im schlauf Joseph/ Inegypten/ und sprach/ stand auf/ niem bin daz kint und sin miter und gang bin Indas erterich ißrael/ fur war sie sind begraben die gesücht haben die sele de $\beta$ kindes/ Joseph stond auf nam daz kind und sein muiter und quam Indaz erterich I Brael [ $5 \mathrm{r}]$. Und als er hort/ das archilaus regiert/ In Juttschem lant/ fur berode sein vatter/ forcht er sich gend da bin/ und ward Im schlauff gewarnt/ und fur Indie teil [?] deß galileschen lands/ quam da hin/ und wonet Inder statt nazareth/ daz erfult wurd/ das gesagt ist durch den propheten/ dann er wurt benent nazarenus.

\section{Markus 1, $32 \mathrm{ff}$.}

Und als aubent ward/ und di sunn under waß gangen/ brachten sie zü Ihne all die übel/ und teufell hetten/ und die gantz stat was gesammelt/ bey der türe/ und er macht fil gesont/ die gereist worden mit fil und mancherley siechtum/ und warf 
fil tenfel herauß/ und verbie $\beta$ sie nit reden mit einander/ [59v] dann si wusten woll das er xstus [= Christus] was/ und am morgen früw vor tag/ ston Jhesus auf/ und geng In die wïsten stat/ und bett dal und Symon und die mit Im waren/ gengen Im nab/ und als sie zu Ibne quamen/ sprachen sie zü Im/ die menschn süchen dich/ und er sprach zui In/ gend wir hin/ In die nechsten gasßen/ und stete/ und daz Ich da predige/ furwar dar umb bin Ich kommen/ und predickt In Iren sammlungen/ und In allem galileschen lande/ und warff auß die teufel/ [...]

\section{Ergebnisse}

Der Beitrag sollte etwas Licht in das Dunkel rund um die als Manuskript 35 in der Leipziger Universitätsbibliothek aufbewahrte vorlutherische deutsche Übersetzung der vier Evangelien bringen. Die bislang nicht sicher datierbare und auch regional nicht klar zuzuordnende Handschrift ist am Ende mit Nicolaus Straub sst. [?] notarius hallensis unterzeichnet. Ein Vergleich dieser Unterschrift mit der Signatur unter notariellen Urkunden, welche der unter anderem in Schwäbisch Hall aktive kaiserliche Notar Nikolaus Straub ausgestellt hat, ergab eindeutig, dass auch die Evangelien-Übersetzung von diesem Notar stammt. Der im Kern süddeutsche Text mit schwäbischem, zum Teil auch fränkischen Einschlag lässt sich im Übrigen sehr gut mit der Vita Straubs in Verbindung bringen: Straub, der um das Jahr 1415 in Leonberg bei Stuttgart geboren sein dürfte, empfing wohl schon früh - vielleicht in einer Klosterschule - die niederen Weihen. Er studierte in Heidelberg, wo er im Mai 1435 das Magisterexamen der Artistenfakultät absolvierte. Später war er Schultheiß in Wimpfen im Tale, um dann den Titel eines kaiserlichen Notars zu erwerben. Als solcher ließ er sich frühestens 1457, vermutlich erst um die Jahreswende 1458/1459 in Schwäbisch Hall nieder. Aller Wahrscheinlichkeit nach 1463, spätestens aber 1465 verließ er die Reichsstadt am Kocher wieder. Somit ist nun eine recht exakte Datierung der Bibelübersetzung möglich, zumal sie eher am Ende von Straubs Zeit in Schwäbisch Hall entstanden sein dürfte, also um 1460/1463.

Straub verfertigte die Übersetzung nach eigenen Angaben ohne Vorlagen und Hilfsmittel, was die - von der Wissenschaft attestierte - hohe Selbständigkeit der Übersetzung erklärt. Soweit einige Kernpassagen dennoch gewisse Ähnlichkeiten mit anderen Übersetzungen - etwa der Luthers - aufweisen, lässt sich dies mit der allgemeinen Bekanntheit der Stellen, einer mündlichen deutschsprachigen Erzähltradition (etwa in Bezug auf die Weihnachtsgeschichte) erklären. Dass die Übersetzung insgesamt als besonders eigenständig gilt, könnte auch mit Straubs Übersetzungstechnik zusammenhängen, denn zweifellos unterscheidet sich die Herangehensweise eines Notars von der eines Geistlichen. So könnte die - bereits Zimmermann aufgefallene ${ }^{161}$ - Reihung von (Teil-) Synonymen als Übersetzung

161 Zimmermann (wie Anm. 1) insbesondere S. 72\%. 
(etwa die Bezeichnung der drei Weisen aus dem Morgenland als die weyßen meister oder kunig bzw. meister und weisen kunig) dem - in der notariellen Arbeit unabdinglichen - Bedürfnis nach Präzision geschuldet sein.

Ungeklärt bleibt Straubs Anlass für die Evangelien-Übersetzung. Möglicherweise verwendete sie Straub, um sich für weitere Dienste zu empfehlen. Obwohl er soweit ersichtlich - stets in weltlichen Ämtern tätig war, zeigt seine Laufbahn eine markante Nähe zur Kirche. Ob es mit seiner Evangelien-Übersetzung in $\mathrm{Zu}$ sammenhang steht, dass Straub in der Folge mit der Reform mehrerer Heilbronner Klöster befasst wurde, bleibt Spekulation. Immerhin empfahl ihn kein geringerer als Abt Johann von Maulbronn, weil Straub der Ding Fliß, ernst und erfarung habe. Straub gelang es, in Heilbronn Karriere zu machen, bald schon begegnet er im - für ihn neu geschaffenen - Amt eines städtischen Generalsyndikus. Aus dem Jahr 1471 stammt ein (ähnlich der Evangelien-Übersetzung) von Straub unterzeichneter lateinischer Kommentar zum römischen Recht anhand der Institutionen Kaiser Justinians. Mehrfach unternahm Straub Dienstreisen im Auftrag der Reichsstadt Heilbronn, so nicht weniger als dreimal nach Rom. Eine Bemerkung in einem 1485 in Rom verfertigten Schreiben an den Heilbronner Rat, in dem Straub um Fürsorge für seine hußfrau bat, zeigt, dass Straub verheiratet war. Vom 11. Mai 1500 stammt Straubs letzte Urkunde, bald darauf dürfte er verstorben sein. 